TRANSACTIONS OF THE

AMERICAN MATHEMATICAL SOCIETY

Volume 361, Number 8, August 2009, Pages 4045-4076

S 0002-9947(09)04732-1

Article electronically published on April 1, 2009

\title{
VALUE FUNCTIONS AND THE DIRICHLET PROBLEM FOR ISAACS EQUATION IN A SMOOTH DOMAIN
}

\author{
JAY KOVATS
}

\begin{abstract}
In this paper, we investigate probabilistic solutions of the Dirichlet problem for the elliptic Isaacs equation in a smooth bounded domain in Euclidean space.
\end{abstract}

\section{Introduction}

We wish to obtain a probabilistic representation for the viscosity solution of the Dirichlet problem for the (possibly degenerate) elliptic Isaacs equation

$$
\left\{\begin{aligned}
\min _{z \in Z} \max _{y \in Y}\{L(y, z, x) v(x)+f(y, z, x)\} & =0 & & \text { in } D, \\
v & =g & & \text { on } \partial D,
\end{aligned}\right.
$$

where $L(y, z, x) u:=\operatorname{tr}\left[a(y, z, x) u_{x x}\right]+b(y, z, x) \cdot u_{x}-c(y, z, x) u, D \subset E_{d}$ is a smooth bounded domain and $g \in C(\bar{D})$. Here, the $d \times d$ matrix $a(y, z, x)$ is nonnegative definite. We assume that our coefficients, $a, b, c, f$ are uniformly continuous, uniformly bounded and Lipschitz continuous in $x$ (uniformly in $y, z$ ), with $c \geq 0 . Y, Z$ are compact metric spaces. There are two Isaacs equations. The upper Isaacs equation $F^{+}$and the lower Isaacs equation $F^{-}$arise in the theory of stochastic differential games (see [ES], $\mathrm{Fr},[\mathrm{I}]$ ) and are defined by

$$
\begin{aligned}
& F^{+}[u]:=\min _{z \in Z} \max _{y \in Y}\left\{\operatorname{tr}\left[a(y, z, x) u_{x x}\right]+b(y, z, x) \cdot u_{x}-c(y, z, x) u+f(y, z, x)\right\}=0, \\
& F^{-}[u]:=\max _{y \in Y} \min _{z \in Z}\left\{\operatorname{tr}\left[a(y, z, x) u_{x x}\right]+b(y, z, x) \cdot u_{x}-c(y, z, x) u+f(y, z, x)\right\}=0 .
\end{aligned}
$$

By our conditions on the coefficients, we know (see IL]) that when the equation in (0.1) is nondegenerate, i.e. $\exists 0<\lambda \leq \Lambda$ for which $\forall y \in Y, z \in Z, x \in D, \lambda I_{d} \leq$ $a(y, z, x) \leq \Lambda I_{d}$, and $\partial D$ satisfies a uniform exterior sphere condition, there exists a unique viscosity solution $v \in C(\bar{D})$ to the Dirichlet problem (0.1). A unique (continuous) viscosity solution also exists in the degenerate elliptic case when $\partial D \in C^{2}$, $\inf _{y, z, x} c(y, z, x) \geq c_{0}>0$ and $\inf _{y, z}\langle a(y, z, x) n(x), n(x)\rangle>0$ on $\partial D$, where $n(x)$ is the outward unit normal to $D$ at $x \in \partial D$. Under the assumption of the existence of an appropriate global barrier, we show that the value functions $v^{+}$and $v^{-}$, given in (0.7) and (0.8), are continuous viscosity solutions for the corresponding Dirichlet problems for $F^{+}$and $F^{-}$, respectively. Hence, in the aforementioned cases where uniqueness is guaranteed, $v^{+}$and $v^{-}$are the continuous viscosity solutions for the corresponding Dirichlet problems. Our results completely cover the nondegenerate

Received by the editors May 7, 2007.

2000 Mathematics Subject Classification. Primary 35B65, 35J60, 49N70, 91A05. 
case, since for uniformly elliptic equations with bounded coefficients, global barriers always exist when $\partial D$ is smooth. (See Assumption 1.0 and the discussion which follows.)

From the viewpoint of partial differential equations, it would be of interest to have an explicit form, especially in the nondegenerate case, for the viscosity solution of (0.1), which is of "Perron-type", as proved by Ishii. Solutions of the nondegenerate Isaacs equation are of interest because (i) any uniformly elliptic equation of the form $F\left(u_{x x}, x\right)=0$ can be shown to be of Isaacs type (see [CC2] (2003)) and (ii) the Isaacs equation is an example of a second-order partial differential equation which is, in general, neither convex nor concave in $u_{x x}$ (i.e., the Isaacs operator $F(m, p, r, x)$ is neither convex nor concave in $m)$. The $C^{2+\alpha}$ regularity theory hasn't been extended to solutions of even the simplest such equations $F\left(u_{x x}\right)=0$, i.e. $F=F(m)$, except in special cases (see [CC2]).

We recall (1982) that the Evans-Krylov theorem states that if $u \in C^{2}(B)$ satisfies the uniformly elliptic equation $F\left(u_{x x}\right)=0$, where $F=F(m)$ is either convex or concave, then $\exists \alpha \in(0,1)$ for which $u \in C_{l o c}^{2+\alpha}(B)$. In 1989, Caffarelli (see CC1]) extended this result to continuous viscosity solutions of $F\left(u_{x x}\right)=0$, where $F$ is either convex or concave in $m$. But what can be said about the viscosity solution of even the simplest equation in $d=3$, i.e.,

$$
\left\{\begin{array}{rlrl}
\Delta v+\left(v_{x^{1} x^{1}}\right)_{+}-\left(v_{x^{2} x^{2}}\right)_{-}=0 & & \text { in } B_{1}, \\
v & =g & & \text { on } \partial B_{1},
\end{array}\right.
$$

for arbitrary fixed smooth $g$ ? This equation is uniformly elliptic and of Isaacs type, since it can be written as

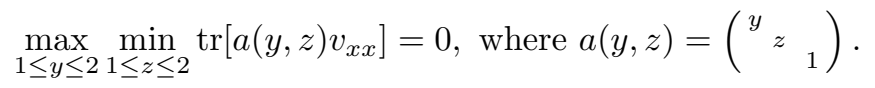

The operator in $(0.3)$ is of the form

$$
\min \left\{\max \left\{L_{1} v, L_{2} v\right\}, \max \left\{L_{3} v, L_{4} v\right\}\right\}
$$

where $L_{1} v=\Delta v=(1,1,1), L_{2} v=(2,1,1), L_{3} v=(1,2,1), L_{4} v=(2,2,1)$ and $n o t$ of the "3-operator" form $\min \left\{\tilde{L}_{1} v, \max \left\{\tilde{L}_{2} v, \tilde{L}_{3} v\right\}\right\}$, to which recent $C^{2+\alpha}$ regularity results (see [CC2]) apply. Any viscosity solution of $\Delta v+\left(v_{x^{1} x^{1}}\right)_{+}-\left(v_{x^{2} x^{2}}\right)_{-}=0$ must be locally $C^{1, \alpha}$ for some $\alpha \in(0,1)$ but what more can be said about these solutions? Since the expression inside the $\max \min$ in $(0.4)$ is an affine function of $y, z$, trivially the Isaaacs condition $\max \min =\min \max$ is satisfied and $(0.3)$ can also be written as $\max \left\{\min \left\{L_{1} v, L_{3} v\right\}, \min \left\{L_{2} v, L_{4} v\right\}\right\}=0$. It is possible that an explicit representation, albeit a probabilistic one, could shed light on regularity properties of solutions. (See $\mathrm{Ka}$ for an explicit representation of viscosity solutions of fully nonlinear degenerate parabolic equations in the whole space.)

From the viewpoint of applications, solutions of Isaacs equations are intuitively thought of as "value" functions of a stochastic differential game of survival between two players, which we loosely describe as follows. We have a probability space $(\Omega, \mathcal{F}, \mathbf{P})$, a filtration of $\sigma$-algebras $\left\{\mathcal{F}_{t}\right\}_{t \geq 0}$ of $\Omega$, complete with respect to $(\mathcal{F}, \mathbf{P})$, and a $d_{1}$-dimensional Wiener process $\left(w_{t}, \mathcal{F}_{t}\right)$ on $(\Omega, \mathcal{F}, \mathbf{P})$. For controls $y, z\left(\mathcal{F}_{t^{-}}\right.$ progressively measurable processes available to players I, II respectively) and $x \in D$, 
we have a solution $x_{t}^{y, z, x}$ of the stochastic equation

$$
x_{t}=x+\int_{0}^{t} \sigma\left(y_{r}, z_{r}, x_{r}\right) d w_{r}+\int_{0}^{t} b\left(y_{r}, z_{r}, x_{r}\right) d r
$$

Here, $\sigma$ is a $d \times d_{1}$ matrix and $b$ is a vector in $E_{d}$, sufficiently smooth to ensure (0.5) has a solution for any $x \in E_{d}$ and $y, z$. For fixed $x \in D$ and choice of controls $y, z$ for the solution of $(0.5)$, we associate a functional

$$
\begin{array}{r}
J(y, z, x):=\mathbf{E}_{x}^{y, z}\left[\int_{0}^{\tau} f\left(y_{r}, z_{r}, x_{r}\right) e^{-\varphi_{r}} d r+g\left(x_{\tau}\right) e^{-\varphi_{\tau}}\right] \\
\text { where } \varphi_{t}^{y, z, x}=\int_{0}^{t} c\left(y_{s}, z_{s}, x_{s}^{y, z, x}\right) d s
\end{array}
$$

$\tau^{y, z, x}=\tau_{D}^{y, z, x}:=\inf \left\{t \geq 0: x_{t}^{y, z, x} \notin D\right\}$, and $x_{t}^{y, z, x}$ is the solution of the stochastic equation (0.5). The idea of the game is that player I chooses controls $y=y_{t}(\omega)$ for $(0.5)$ in order to maximize $J$, while player II chooses controls $z=z_{t}(\omega)$ for $(0.5)$ to minimize $J$. Each player "moves", step-by-step, in fixed time intervals, into which the lifetime of the game is divided. There are two values for this game: an upper value (of $J$ ) in which player I (the maximizer) has the advantage, and a lower value in which player II (the minimizer) has the advantage. By "advantage", we mean that the player knows how his opponent has moved, before he moves. Games of survival in which $f \geq 0$ and $g \equiv 0$ are called generalized pursuit-evasion games, the most famous of which is the pursuit-evasion game: $f \equiv 1, c \equiv 0$. In this case, $J(y, z, x)=\mathbf{E} \tau^{y, z, x}$, the mean exit-time or capture time of the process $x_{t}^{y, z, x}$ from the domain $D$. Here, player I, wishing to maximize the mean capture time, is the evader, while player II, wanting to minimize the mean capture time, is the pursuer. See $[\mathrm{Fr}]$ for a rigorous treatment of deterministic, nonstochastic differential games.

Definition 0.1. An admissible control for player I (respectively II) is an $\mathcal{F}_{t^{-}}$ progressively measurable function $y_{t}(\omega)$ (respectively $z_{t}(\omega)$ ), defined on $[0, \infty) \times \Omega$, with values in $Y$ (respectively $Z$ ). The set of all admissible controls for player I (respectively II) is denoted by $\mathcal{M}$ (respectively $\mathcal{N}$ ). Controls $y^{1}, y^{2} \in \mathcal{M}$ are said to be equal on $[s, t]$ if $\mathbf{P}\left\{\left|y_{r}^{1}-y_{r}^{2}\right|=0\right.$, a.e. $\left.r \in[s, t]\right\}=1$. An analogous statement holds for controls in $\mathcal{N}$.

Definition 0.2. An admissible strategy for player I (respectively II) is a mapping $\alpha: \mathcal{N} \rightarrow \mathcal{M}$ (respectively $\beta: \mathcal{M} \rightarrow \mathcal{N}$ ) which preserves the indistinguishability of controls. That is, if $z^{1}, z^{2} \in \mathcal{N}$ and $z^{1}=z^{2}$ on $[s, t]$, then $\alpha\left(z^{1}\right)=\alpha\left(z^{2}\right)$ on $[s, t]$. The set of all admissible strategies for player I (respectively II) is denoted by $\Gamma$ (respectively $\Delta$ ).

Definition 0.3. We define the upper value $v^{+}$of the differential game by

$$
\begin{aligned}
v^{+}(x) & :=\sup _{\alpha \in \Gamma} \inf _{z \in \mathcal{N}} J(\alpha(z), z, x) \\
& =\sup _{\alpha \in \Gamma} \inf _{z \in \mathcal{N}} \mathbf{E}_{x}^{\alpha, z}\left[\int_{0}^{\tau} f\left(\alpha(z)_{r}, z_{r}, x_{r}\right) e^{-\varphi_{r}} d r+g\left(x_{\tau}\right) e^{-\varphi_{\tau}}\right] .
\end{aligned}
$$


and the lower value $v^{-}$of the differential game by

$$
\begin{aligned}
v^{-}(x) & :=\inf _{\beta \in \Delta} \sup _{y \in \mathcal{M}} J(y, \beta(y), x) \\
& =\inf _{\beta \in \Delta} \sup _{y \in \mathcal{M}} \mathbf{E}_{x}^{y, \beta}\left[\int_{0}^{\tau} f\left(y_{r}, \beta(y)_{r}, x_{r}\right) e^{-\varphi_{r}} d r+g\left(x_{\tau}\right) e^{-\varphi_{\tau}}\right] .
\end{aligned}
$$

In the special case $\tau=+\infty, g \equiv 0$ and the lower bound for $c$ is appropriately large, it follows from [FS] that both $v^{+}$and $v^{-}$satisfy a dynamic programming principle. From this it can be shown that $v^{+}$is the unique bounded viscosity solution of the upper Isaacs equation $F^{+}[v]=0$ in $E_{d}$ and $v^{-}$is the unique bounded viscosity solution of the lower Isaacs equation $F^{-}[v]=0$ in $E_{d}$. Hence if the Isaacs condition holds, i.e. $F^{+}=F^{-}$, we immediately have that $v^{+}=v^{-}$, in which case the differential game is said to have value. (See also Święch [S], 1996.) In [FS] (1988), Fleming and Souganidis examined probabilistic expressions for the viscosity solution of the Cauchy problem for the parabolic Isaacs equation in a strip $H_{T}:=[0, T) \times E_{d}$, with terminal data function $g(x)$ and discount factor $c \equiv 0$, where the coefficients depended on $t$ and $x$ (as well as controls $y, z$ ). They showed that if $f$ and the coefficients of the process are bounded, uniformly continuous, Lipschitz in $(t, x)$, and $g \in C_{b}^{0,1}\left(E_{d}\right)$, then the upper value function

$v(t, x)=\sup _{\alpha \in \Gamma(t)} \inf _{z \in \mathcal{N}(t)} \mathbf{E}_{t, x}^{\alpha, z}\left[\int_{t}^{T} f\left(r, x_{r}, \alpha(z)_{r}, z_{r}\right) d r+g\left(x_{T}\right)\right], \quad 0 \leq t \leq T, x \in E_{d}$

is the unique viscosity solution of the Cauchy problem: $F^{+}[u]+u_{t}=0$ in $H_{T}, u(T, x)$ $=g(x), x \in E_{d}$, and satisfies the dynamic programming principle: $\forall s$ with $t \leq s \leq$ $T$,

$$
v(t, x)=\sup _{\alpha \in \Gamma(t)} \inf _{z \in \mathcal{N}(t)} \mathbf{E}_{t, x}^{\alpha, z}\left[\int_{t}^{s} f\left(r, x_{r}, \alpha(z)_{r}, z_{r}\right) d r+v\left(s, x_{s}\right)\right] .
$$

Here, for $t \leq s \leq T, x_{s}^{\alpha(z), z}(t, x)$ is the solution of the stochastic differential equation

$$
x_{s}=x+\int_{t}^{s} \sigma\left(r, x_{r}, y_{r}, z_{r}\right) d w_{r}+\int_{t}^{s} b\left(r, x_{r}, y_{r}, z_{r}\right) d r
$$

with control $y=\alpha(z)$, while $\Gamma(t), \mathcal{N}(t)$ denote, respectively, strategies for player I and controls for player II, defined on $[t, T]$. Corresponding statements hold for the lower value function and the lower Isaacs equation.

If we define $S_{t, T} g(x)=v(t, x)$, the dynamic programming principle (0.10) is the statement that for $0 \leq t \leq s \leq T, S_{t, T}=S_{t, s} \circ S_{s, T}$ as operators on $C_{b}^{0,1}\left(E_{d}\right)$. If the coefficients of our process and $f$ are independent of $t$, and our process is at the point $x$ at the initial time $t=0,(0.9)$ can be rewritten as

$v(t, x)=S_{0, T-t} g(x)=\sup _{\alpha \in \Gamma(0, T)} \inf _{z \in \mathcal{N}(0, T)} \mathbf{E}_{0, x}^{\alpha, z}\left[\int_{0}^{T-t} f\left(\alpha(z)_{r}, z_{r}, x_{r}\right) d r+g\left(x_{T-t}\right)\right]$.

That is, the right-hand side depends, for $t \in[0, T]$, only on differences $T-t$. Setting $Q(T-t) g(x):=S_{0, T-t} g(x),(0.10)$ reads: for $t \leq s \leq T, Q(T-t)=Q(s-t) \circ$ $Q(T-s)$. Since $s-t, T-s \in[0, T]$ were arbitrary, we have $Q\left(t_{1}+t_{2}\right)=Q\left(t_{1}\right) \circ Q\left(t_{2}\right)$ for any $t_{1}, t_{2} \in[0, T]$ such that $t_{1}+t_{2} \in[0, T]$. That is, the family of operators 
$\{Q(t)\}$ forms a nonlinear semigroup on $C_{b}^{0,1}\left(E_{d}\right)$, where

$$
Q(t) g(x):=\sup _{\alpha \in \Gamma} \inf _{z \in \mathcal{N}} \mathbf{E}_{x}^{\alpha, z}\left[\int_{0}^{t} f\left(\alpha(z)_{r}, z_{r}, x_{r}\right) d r+g\left(x_{t}\right)\right]
$$

and $\Gamma, \mathcal{N}$ denote, respectively, stategies for player 1 and controls for player 2 on $[0, T]$.

It also follows from Nisio's results in $\mathbb{N} 1$ that $Q(t) g$ is a semigroup on $C_{b}\left(E_{d}\right)$ (bounded, uniformly continuous functions on $E_{d}$ ). In 1988, M. Nisio (see [N1) studied probabilistic solutions for the Cauchy problem for the parabolic Isaacs equation with coefficients independent of $t$. The expressions for $v^{+}(t, x)$ and $v^{-}(t, x)$ in [N1, however, differ from those found in [FS], as the notion of strategy is not made explicit. Actually, the [FS] results use a discretization technique very similar to that used in [N1. Loosely stated, in the definition of the upper value function from [N1, player I can freely choose controls (to maximize $J$ ) in a given time interval, while player II can choose only among constant controls in that interval (to minimize $J$ ). Similarly, for the lower value function, player II can freely choose controls, while player I can use only constant controls. So, in a sense, the notion of strategy is implicitly built into the game. Under the Isaacs condition $F^{+}=F^{-}$, Nisio showed that both $v^{+}(t, x)$ and $v^{-}(t, x)$ are viscosity solutions of the Cauchy problem for the Isaacs equation: $F[v]-v_{t}=0$ in $H_{T}, v(0, x)=g(x), x \in E_{d}$.

In 1993, Fleming and Nisio (see [FN]) examined value functions and min-max equations associated with a stochastic differential game in a Hilbert space, with dynamics governed by the controlled (time-homogeneous) Zakai equation. We refer the reader to $\S \S 5$ and 6 , wherein the authors use a time-homogeneous analogue of $\S 2$ in FS to prove a dynamic programming principle for the corresponding value functions. Our formulation of value functions is consistent with that of both [FN] and $[\mathrm{N} 1$.

Under the assumptions that (i) $D \subset E_{d}$ is a domain for which there exists a global barrier relative to $L^{0}(y, z, x)$ (Assumption 1.0), and (ii) the coefficients of the diffusion process $(0.5)$ are uniformly bounded and Lipschitz, $\left((1.1)^{\prime}\right)$, we show that the following forms a nonlinear semigroup on $C(\bar{D})$ :

$$
J(t) g(x)=\sup _{\alpha \in \Gamma} \inf _{z \in \mathcal{N}} \mathbf{E}_{x}^{\alpha, z}\left[\int_{0}^{t \wedge \tau} f\left(\alpha(z)_{r}, z_{r}, x_{r}\right) e^{-\varphi_{r}} d r+g\left(x_{t \wedge \tau}\right) e^{-\varphi_{t \wedge \tau}}\right]
$$

where for $x \in D, \alpha \in \Gamma$ and $z \in \mathcal{N}, \tau=\tau^{\alpha(z), z, x}$ is the first exit time of the process $x_{t}^{\alpha(z), z, x}$ from $D$, and $x_{t}^{\alpha(z), z, x}$ is the solution of the stochastic differential equation (0.5) with $y=\alpha(z)$. From this fact it follows (see Theorem 1.4, the last remark in $\S 1$ and (1.6)) that $v^{+}$in (0.7) satisfies a dynamic programming principle, which in turn implies that $v^{+}$is a continuous viscosity solution of the upper Issacs equation in $D$ satisfying $v=g$ on $\partial D$. The same holds for $v^{-}$in (0.8) and the lower Isaacs equation.

\section{Continuity properties of $J(t, x, y, z)$ And Related FunCtionals}

In this section, we establish continuity properties of the functional

$$
J(t, x, y, z)=\mathbf{E}_{x}^{y, z}\left[\int_{0}^{t \wedge \tau} f\left(y_{r}, z_{r}, x_{r}\right) e^{-\varphi_{r}} d r+g\left(x_{t \wedge \tau}\right) e^{-\varphi_{t \wedge \tau}}\right]
$$


where $x \in D, y \in \mathcal{M}, z \in \mathcal{N}$ and $g \in C_{b}(D)$. For simplicity, we assume that $t \in[0, T]$. In the case $\tau^{y, z, x}=+\infty$, corresponding to $D=E_{d}$, it is a straightforward matter to show that $J$ is uniformly continuous on $[0, T] \times E_{d} \times \mathcal{M} \times \mathcal{N}$ (see Proposition 2.2 in N1]). Continuity and semigroup properties of the Bellman functional (only one player) are established for the case $D=E_{d}$ in $\S \S 3.1-3.3$ of K1], $\S \S 5.1,5.2$ of $\mathrm{BL}$ and $\S \S 1,2$ of [N2]. Continuity and semigroup properties of the Bellman functional for nondegenerate processes in a smooth, bounded domain $D$ are addressed in $\S 2$ of [LM1. In general, for the case $D \neq E_{d}, J$ will not be continuous in $x$ even in the linear (0-player) case. To offset this, we assume the existence of a global barrier (see Assumption 1.0 below).

We define a distance function on $\mathcal{M}$, by $\tilde{\rho}_{\mathcal{M}}\left(y^{1}, y^{2}\right)=\mathbf{E} \int_{0}^{T} \rho_{Y}\left(y_{r}^{1}, y_{r}^{2}\right) d r$, for $y^{1}, y^{2} \in \mathcal{M}$, where $\rho_{Y}$ is the metric on $Y$. We may always assume that $\rho_{Y}<1$, since otherwise we use the metric given by $\rho_{Y}^{\prime}:=\frac{2}{\pi} \tan ^{-1}\left(\rho_{Y}\right)$. We define a distance function on $\mathcal{N}$ in the same way. Fix $t \in[0, T], x \in E_{d}$. We assume that for $y, y^{1}, y^{2} \in Y, z, z^{1}, z^{2} \in Z, x, x^{1}, x^{2} \in E_{d}$,

$$
\begin{aligned}
\left|h\left(y^{1}, z^{1}, x^{1}\right)-h\left(y^{2}, z^{2}, x^{2}\right)\right| & \leq C\left\{\left|x^{1}-x^{2}\right|+\rho_{Y}\left(y^{1}, y^{2}\right)+\rho_{Z}\left(z^{1}, z^{2}\right)\right\} \\
|h(y, z, x)| & \leq C
\end{aligned}
$$

for $h=\sigma, b, c, f$. Then for $y^{1}, y^{2} \in \mathcal{M}, z^{1}, z^{2} \in \mathcal{N}, x^{1}, x^{2} \in E_{d}$, Itô's formula yields for $t \in[0, T]$,

$$
\begin{aligned}
& \mathbf{E}\left|x_{t}^{y^{1}, z^{1}, x^{1}}-x_{t}^{y^{2}, z^{2}, x^{2}}\right|^{2} \leq\left|x^{1}-x^{2}\right|^{2} \\
& +\left(3 C^{2}+4 C\right) \mathbf{E} \int_{0}^{t}\left\{\left|x_{r}^{y^{1}, z^{1}, x^{1}}-x_{r}^{y^{2}, z^{2}, x^{2}}\right|^{2}+\rho_{Y}^{2}\left(y_{r}^{1}, y_{r}^{2}\right)+\rho_{Z}^{2}\left(z_{r}^{1}, z_{r}^{2}\right)\right\} d r \\
& \quad \leq\left|x^{1}-x^{2}\right|^{2} \\
& +\left(3 C^{2}+4 C\right)\left\{\int_{0}^{t} \mathbf{E}\left|x_{r}^{y^{1}, z^{1}, x^{1}}-x_{r}^{y^{2}, z^{2}, x^{2}}\right|^{2} d r+\tilde{\rho}_{\mathcal{M}}\left(y^{1}, y^{2}\right)+\tilde{\rho}_{\mathcal{N}}\left(z^{1}, z^{2}\right)\right\} .
\end{aligned}
$$

Hence Gronwall's inequality gives, for any $t \in[0, T]$,

$$
\mathbf{E}\left|x_{t}^{y^{1}, z^{1}, x^{1}}-x_{t}^{y^{2}, z^{2}, x^{2}}\right|^{2} \leq C_{2} e^{C_{2} t}\left\{\left|x^{1}-x^{2}\right|^{2}+\tilde{\rho}_{\mathcal{M}}\left(y^{1}, y^{2}\right)+\tilde{\rho}_{\mathcal{N}}\left(z^{1}, z^{2}\right)\right\},
$$

where $C_{2}>1$ is a constant, depending only on $C$. This estimate, along with the BDG inequality implies

$$
\mathbf{E} \sup _{0 \leq t \leq T}\left|x_{t}^{y^{1}, z^{1}, x^{1}}-x_{t}^{y^{2}, z^{2}, x^{2}}\right|^{2} \leq C_{4} e^{C_{4} T}\left\{\left|x^{1}-x^{2}\right|^{2}+\tilde{\rho}_{\mathcal{M}}\left(y^{1}, y^{2}\right)+\tilde{\rho}_{\mathcal{N}}\left(z^{1}, z^{2}\right)\right\},
$$

where $C_{4}$ depends only on $C$. Define $L^{0}(y, z, x) u(x):=\operatorname{tr}\left[a(y, z, x) u_{x x}(x)\right]+$ $b(y, z, x) \cdot u_{x}(x)$. Our main assumption throughout this paper is

Assumption 1.0. $\exists \psi \in C^{2}(\bar{D})$ such that $\forall y \in Y, z \in Z, L^{0}(y, z) \psi \leq-1$ in $D, \psi \equiv 0$ on $\partial D$.

Observe that in the nondegenerate case, that is, $\lambda I_{d} \leq a(y, z, x) \leq \Lambda I_{d}$, a uniform global barrier always exists if $\partial D \in C^{2}$. For example, if $D=B_{r}\left(x_{0}\right)$, an explicit 
$C^{\infty}(D)$ global barrier is given by $\psi(x)=\cosh (k r)-\cosh \left(k\left|x-x_{0}\right|\right)$, for $k$ sufficiently large. More generally, if $\partial D$ satisfies a uniform exterior sphere condition, then the solution $\psi \in C^{2+\alpha}(\bar{D})$ of the Dirichlet problem for the convex Bellman-Pucci equation

$$
\left\{\begin{aligned}
\mathcal{M}^{+}\left(\psi_{x x}, \lambda, \Lambda\right)+C\left|\psi_{x}\right|+1 & =0 & & \text { in } D \\
\psi & =0 & & \text { on } \partial D,
\end{aligned}\right.
$$

where $C$ is as in $(1.1)^{\prime}$, will be a global barrier with respect to $L^{0}(y, z)$ on $D$. This follows immediately from our assumption $\lambda I_{d} \leq a(y, z, x) \leq \Lambda I_{d}$, and the definition of $\mathcal{M}^{+}$. Note that we can express the above pde as

$$
\sup _{(a, b) \in \mathcal{A}_{\lambda, \Lambda} \times \bar{B}_{1}(0)}\left\{\operatorname{tr}\left[a \psi_{x x}\right]+C b \cdot \psi_{x}\right\}+1=0,
$$

where $\mathcal{A}_{\lambda, \Lambda}$ denotes the set of symmetric $d \times d$ symmetric matrices with eigenvalues in $[\lambda, \Lambda]$. (See pp. 14-15 in CC1 for the definition of $\mathcal{M}^{+}$.)

In the degenerate case, global barriers occur in certain cases. For example, say $D:=\left\{x \in E_{d}: \psi(x)>0\right\}$, where $\psi$ is $C^{2}\left(E_{d}\right)$ and uniformly concave, with $\psi_{x x} \leq-\kappa I_{d}$, for some $\kappa>0$. If $\operatorname{tr}[a(y, z, x)] \geq \Lambda(x)>\frac{1}{\kappa}$, and $\left|\psi_{x}(x)\right| \leq \frac{\kappa \Lambda(x)-1}{C}$, where $C$ is as in $(1.1)^{\prime}$, then $\psi$ will be a barrier for $L^{0}$ on $D$, since

$$
L^{0}(y, z, x) \psi \leq-\kappa \operatorname{tr}[a(y, z, x)]+|b(y, z, x)|\left|\psi_{x}(x)\right| \leq-\kappa \Lambda(x)+C\left|\psi_{x}(x)\right| \leq-1 .
$$

Of course, here we've assumed that $a(y, z, x)$ has at least one positive eigenvalue in $D$.

The following is a variation of a lemma due to M. Safonov.

Lemma 1.1. For $k=1,2$ and $x_{t}^{k}:=x_{t}^{y^{k}, z^{k}, x^{k}}$, let $\tau^{k}$ be the first exit time of $x_{t}^{k}$ from the open set $D$. Then for a finite constant $K=K(\psi)$,

$$
\mathbf{E}\left|\tau^{1} \wedge T-\tau^{2} \wedge T\right| \leq K \cdot \mathbf{E} \sup _{0 \leq t \leq T}\left|x_{t}^{1}-x_{t}^{2}\right| .
$$

Proof. Define $\tau:=\tau^{1} \wedge \tau^{2}$. Note that $\psi \geq 0$ in $D$. By Ito's formula,

$$
\begin{aligned}
\mathbf{E}\left(\tau^{k} \wedge T-\tau \wedge T\right) & =\mathbf{E} \int_{\tau \wedge T}^{\tau^{k} \wedge T} 1 d t \leq-\mathbf{E} \int_{\tau \wedge T}^{\tau^{k} \wedge T} L_{t}^{k} \psi\left(x_{t}^{k}\right) d t \\
& =\mathbf{E}\left[\psi\left(x_{\tau \wedge T}^{k}\right)-\psi\left(x_{\tau^{k} \wedge T}^{k}\right)\right] \leq \mathbf{E} I_{\left\{\tau<T, \tau \neq \tau^{k}\right\}} \psi\left(x_{\tau}^{k}\right) .
\end{aligned}
$$

On the set $\left\{\tau<T, \tau \neq \tau^{k}\right\}$, we have $\psi\left(x_{\tau}^{k}\right)=\left|\psi\left(x_{\tau}^{1}\right)-\psi\left(x_{\tau}^{2}\right)\right|$. Moreover, since $\tau=\tau^{1} \wedge \tau^{2}$, the sets $\left\{\tau \neq \tau^{1}\right\}$ and $\left\{\tau \neq \tau^{2}\right\}$ are disjoint. Therefore,

$$
\begin{aligned}
\mathbf{E}\left|\tau^{1} \wedge T-\tau^{2} \wedge T\right| & =\sum_{k=1}^{2} \mathbf{E}\left(\tau^{k} \wedge T-\tau \wedge T\right) \leq \mathbf{E} I_{\{\tau<T\}}\left|\psi\left(x_{\tau}^{1}\right)-\psi\left(x_{\tau}^{2}\right)\right| \\
& \leq K \cdot \mathbf{E} I_{\{\tau<T\}}\left|x_{\tau}^{1}-x_{\tau}^{2}\right| \leq K \cdot \mathbf{E} \sup _{0 \leq t \leq T}\left|x_{t}^{1}-x_{t}^{2}\right|,
\end{aligned}
$$

where

$$
K:=\sup _{x \in D, y \in \partial D} \frac{|\psi(x)-\psi(y)|}{|x-y|}=\sup _{x \in D} \frac{\psi(x)}{\operatorname{dist}(x, \partial D)}<\infty .
$$

This lemma and estimate (1.3) immediately give

$$
\begin{aligned}
& \mathbf{E}\left|\tau^{y^{1}, z^{1}, x^{1}} \wedge T-\tau^{y^{2}, z^{2}, x^{2}} \wedge T\right| \\
& \quad \leq K \sqrt{C_{4}} e^{C_{4} T}\left\{\left|x^{1}-x^{2}\right|+\sqrt{\tilde{\rho}_{\mathcal{M}}\left(y^{1}, y^{2}\right)}+\sqrt{\tilde{\rho}_{\mathcal{N}}\left(z^{1}, z^{2}\right)}\right\} .
\end{aligned}
$$


Theorem 1.2. For $g \in C_{b}(D)$, the function $J(t, x, y, z)$ in (1) is uniformly continuous on $[0, T] \times D \times \mathcal{M} \times \mathcal{N}$.

Proof. First, we show uniform continuity on $D \times \mathcal{M} \times \mathcal{N}$. So fix $t \in[0, T], x^{1}, x^{2} \in$ $D, y^{1}, y^{2} \in \mathcal{M}, z^{1}, z^{2} \in \mathcal{N}$. For convenience, we use the following notation: for $k=1,2$, we set $\tau^{k}=\tau^{y^{k}, z^{k}, x^{k}}, x_{t}^{k}=x_{t}^{y^{k}, z^{k}, x^{k}}, \varphi_{t}^{k}=\int_{0}^{t} c\left(y_{r}^{k}, z_{r}^{k}, x_{r}^{k}\right) d r$. Then

$$
\begin{aligned}
& J\left(t, x^{1}, y^{1}, z^{1}\right)-J\left(t, x^{2}, y^{2}, z^{2}\right) \\
& =\mathbf{E}\left[\int_{0}^{t \wedge \tau^{1}} f\left(y_{r}^{1}, z_{r}^{1}, x_{r}^{1}\right) e^{-\varphi_{r}^{1}} d r-\int_{0}^{t \wedge \tau^{2}} f\left(y_{r}^{2}, z_{r}^{2}, x_{r}^{2}\right) e^{-\varphi_{r}^{2}} d r\right] \\
& \quad+\mathbf{E}\left[g\left(x_{t \wedge \tau^{1}}^{1}\right) e^{-\varphi_{t \wedge \tau^{1}}^{1}}-g\left(x_{t \wedge \tau^{2}}^{2}\right) e^{-\varphi_{t \wedge \tau^{2}}^{2}}\right] \\
& :=J_{1}+J_{2},
\end{aligned}
$$

where

$$
\begin{aligned}
\left|J_{1}\right| \leq & \mathbf{E}\left|\int_{0}^{t \wedge \tau^{1}} f\left(y_{r}^{1}, z_{r}^{1}, x_{r}^{1}\right) e^{-\varphi_{r}^{1}} d r-\int_{0}^{t \wedge \tau^{2}} f\left(y_{r}^{2}, z_{r}^{2}, x_{r}^{2}\right) e^{-\varphi_{r}^{2}} d r\right| \\
\leq & \mathbf{E} \int_{0}^{t \wedge \tau^{1}}\left|f\left(y_{r}^{1}, z_{r}^{1}, x_{r}^{1}\right) e^{-\varphi_{r}^{1}}-f\left(y_{r}^{2}, z_{r}^{2}, x_{r}^{2}\right) e^{-\varphi_{r}^{2}}\right| d r \\
& +\mathbf{E}\left|\int_{t \wedge \tau^{2}}^{t \wedge \tau^{1}} f\left(y_{r}^{2}, z_{r}^{2}, x_{r}^{2}\right) e^{-\varphi_{r}^{2}} d r\right| \\
\leq & \|f\|_{\infty} \int_{0}^{t} \mathbf{E}\left|e^{-\varphi_{r}^{1}}-e^{-\varphi_{r}^{2}}\right| d r+\mathbf{E} \int_{0}^{t}\left|f\left(y_{r}^{1}, z_{r}^{1}, x_{r}^{1}\right)-f\left(y_{r}^{2}, z_{r}^{2}, x_{r}^{2}\right)\right| d r \\
& +\|f\|_{\infty} \mathbf{E}\left|t \wedge \tau^{1}-t \wedge \tau^{2}\right| .
\end{aligned}
$$

By the Mean Value Theorem,

$$
\begin{aligned}
\left|e^{-\varphi_{r}^{1}}-e^{-\varphi_{r}^{2}}\right| & \leq\left|\varphi_{r}^{1}-\varphi_{r}^{2}\right| \\
& \leq \int_{0}^{r}\left|c\left(y_{s}^{1}, z_{s}^{1}, x_{s}^{1}\right)-c\left(y_{s}^{2}, z_{s}^{2}, x_{s}^{2}\right)\right| d s \\
& \leq C \int_{0}^{r}\left\{\rho_{Y}\left(y_{s}^{1}, y_{s}^{2}\right)+\rho_{Z}\left(z_{s}^{1}, z_{s}^{2}\right)+\left|x_{s}^{1}-x_{s}^{2}\right|\right\} d s .
\end{aligned}
$$

Similarly

$$
\int_{0}^{t}\left|f\left(y_{r}^{1}, z_{r}^{1}, x_{r}^{1}\right)-f\left(y_{r}^{2}, z_{r}^{2}, x_{r}^{2}\right)\right| d r \leq C \int_{0}^{t}\left\{\rho_{Y}\left(y_{r}^{1}, y_{r}^{2}\right)+\rho_{Z}\left(z_{r}^{1}, z_{r}^{2}\right)+\left|x_{r}^{1}-x_{r}^{2}\right|\right\} d r .
$$

From inequalities $(1.2),(1.4)$, the definitions of $\tilde{\rho}_{\mathcal{M}}, \tilde{\rho}_{\mathcal{N}}$, and the fact that $\tilde{\rho}_{\mathcal{M}} \leq T$, we have

$$
\left|J_{1}\right| \leq e^{C_{6} T} N_{7}\left\{\left|x^{1}-x^{2}\right|+\sqrt{\tilde{\rho}_{\mathcal{M}}\left(y^{1}, y^{2}\right)}+\sqrt{\tilde{\rho}_{\mathcal{N}}\left(z^{1}, z^{2}\right)}\right\}
$$


where $N_{7}=N_{7}(K, C)$, and $C_{6}$ depends only on $C$. Splitting up $J_{2}$ in the usual way, and again using the Mean Value Theorem, we get

$$
\begin{gathered}
\left|J_{2}\right| \leq\|g\|_{\infty} \mathbf{E}\left|\varphi_{t \wedge \tau^{1}}^{1}-\varphi_{t \wedge \tau^{2}}^{2}\right|+\mathbf{E}\left|g\left(x_{t \wedge \tau^{1}}^{1}\right)-g\left(x_{t \wedge \tau^{2}}^{2}\right)\right| \\
\left|\varphi_{t \wedge \tau^{1}}^{1}-\varphi_{t \wedge \tau^{2}}^{2}\right| \leq\left|\varphi_{t \wedge \tau^{1}}^{1}-\varphi_{t \wedge \tau^{2}}^{1}\right|+\left|\varphi_{t \wedge \tau^{2}}^{1}-\varphi_{t \wedge \tau^{2}}^{2}\right| \\
\leq C\left|t \wedge \tau^{1}-t \wedge \tau^{2}\right|+C \int_{0}^{t \wedge \tau^{2}}\left\{\rho_{Y}\left(y_{r}^{1}, y_{r}^{2}\right)+\rho_{Z}\left(z_{r}^{1}, z_{r}^{2}\right)+\left|x_{r}^{1}-x_{r}^{2}\right|\right\} d r .
\end{gathered}
$$

By inequality (1.4), we get, for some constant $N_{8}=N_{8}(K, C)$,

$$
\mathbf{E}\left|\varphi_{t \wedge \tau^{1}}^{1}-\varphi_{t \wedge \tau^{2}}^{2}\right| \leq e^{C_{5} T} N_{8}\left\{\left|x^{1}-x^{2}\right|+\sqrt{\tilde{\rho}_{\mathcal{M}}\left(y^{1}, y^{2}\right)}+\sqrt{\tilde{\rho}_{\mathcal{N}}\left(z^{1}, z^{2}\right)}\right\} .
$$

Fix $\varepsilon>0$. Since $g \in C_{b}(D), \exists \delta(\varepsilon)>0$ such that for $\xi, \xi^{\prime} \in D,\left|\xi-\xi^{\prime}\right|<\delta(\varepsilon)$ implies $\left|g(\xi)-g\left(\xi^{\prime}\right)\right|<\varepsilon$. Then

$$
\mathbf{E}\left|g\left(x_{t \wedge \tau^{1}}^{1}\right)-g\left(x_{t \wedge \tau^{2}}^{2}\right)\right| \leq 2\|g\|_{\infty} \mathbf{P}\left\{\left|x_{t \wedge \tau^{1}}^{1}-x_{t \wedge \tau^{2}}^{2}\right| \geq \delta\right\}+\varepsilon .
$$

Writing $\left|x_{t \wedge \tau^{1}}^{1}-x_{t \wedge \tau^{2}}^{2}\right| \leq\left|x_{t \wedge \tau^{1}}^{1}-x_{t \wedge \tau^{2}}^{1}\right|+\left|x_{t \wedge \tau^{2}}^{1}-x_{t \wedge \tau^{2}}^{2}\right|$, the BDG inequality and inequality (1.4) yield

$$
\begin{aligned}
& \mathbf{E}\left|x_{t \wedge \tau^{1}}^{1}-x_{t \wedge \tau^{2}}^{1}\right| \leq N C \mathbf{E}_{x} \sqrt{\left|t \wedge \tau^{1}-t \wedge \tau^{2}\right|}+C \mathbf{E}\left|t \wedge \tau^{1}-t \wedge \tau^{2}\right| \\
& \leq N C \sqrt{\mathbf{E}\left|t \wedge \tau^{1}-t \wedge \tau^{2}\right|}+C \mathbf{E}\left|t \wedge \tau^{1}-t \wedge \tau^{2}\right| \\
& \leq N_{3} e^{C_{4} T}\left\{\left|x^{1}-x^{2}\right|^{1 / 2}+\sqrt[4]{\tilde{\rho}_{\mathcal{M}}\left(y^{1}, y^{2}\right)}+\sqrt[4]{\tilde{\rho}_{\mathcal{N}}\left(z^{1}, z^{2}\right)}\right. \\
&\left.\quad+\left|x^{1}-x^{2}\right|+\sqrt{\tilde{\rho}_{\mathcal{M}}\left(y^{1}, y^{2}\right)}+\sqrt{\tilde{\rho}_{\mathcal{N}}\left(z^{1}, z^{2}\right)}\right\},
\end{aligned}
$$

where $N_{3}=N_{3}(K, C)$. By (1.3),

$$
\begin{aligned}
\mathbf{E}\left|x_{t \wedge \tau^{2}}^{1}-x_{t \wedge \tau^{2}}^{2}\right| & \leq \mathbf{E} \sup _{0 \leq s \leq T}\left|x_{s}^{1}-x_{s}^{2}\right| \\
& \leq \sqrt{C_{4}} e^{C_{4} T}\left\{\left|x^{1}-x^{2}\right|+\sqrt{\tilde{\rho}_{\mathcal{M}}\left(y^{1}, y^{2}\right)}+\sqrt{\tilde{\rho}_{\mathcal{N}}\left(z^{1}, z^{2}\right)}\right\} .
\end{aligned}
$$

Thus, since $\tilde{\rho} \leq T$ (since $\rho<1$ ), we get, for some $N_{4}=N_{4}(K, C)$,

$$
\begin{aligned}
\mathbf{E}\left|x_{t \wedge \tau^{1}}^{1}-x_{t \wedge \tau^{2}}^{2}\right| \leq & N_{4}\left(1+\sqrt{\operatorname{diam}(D)}+T^{1 / 4}\right) e^{C_{4} T}\left\{\left|x^{1}-x^{2}\right|^{1 / 2}+\sqrt[4]{\tilde{\rho}_{\mathcal{M}}\left(y^{1}, y^{2}\right)}\right. \\
& \left.+\sqrt[4]{\tilde{\rho}_{\mathcal{N}}\left(z^{1}, z^{2}\right)}\right\} \\
= & N_{6}\left\{\left|x^{1}-x^{2}\right|^{1 / 2}+\sqrt[4]{\tilde{\rho}_{\mathcal{M}}\left(y^{1}, y^{2}\right)}+\sqrt[4]{\tilde{\rho}_{\mathcal{N}}\left(z^{1}, z^{2}\right)}\right\}
\end{aligned}
$$

where $N_{6}=N_{6}(C, D, K, T)$. By Chebyshev's inequality,

$$
\mathbf{P}\left\{\left|x_{t \wedge \tau^{1}}^{1}-x_{t \wedge \tau^{2}}^{2}\right| \geq \delta\right\} \leq \frac{N_{6}}{\delta}\left\{\left|x^{1}-x^{2}\right|^{1 / 2}+\sqrt[4]{\tilde{\rho}_{\mathcal{M}}\left(y^{1}, y^{2}\right)}+\sqrt[4]{\tilde{\rho}_{\mathcal{N}}\left(z^{1}, z^{2}\right)}\right\},
$$

and hence, for $t \in[0, T]$, and some constant $N_{9}=N_{9}\left(C, K, \mid g \|_{\infty}\right)$, we get

$$
\begin{array}{r}
\left|J_{1}+J_{2}\right| \leq\left[N_{9} e^{C_{7} T}\left(\sqrt{\operatorname{diam}(D)} \vee T^{1 / 4}\right)+\frac{2\|g\|_{\infty} N_{6}}{\delta}\right]\left\{\left|x^{1}-x^{2}\right|^{1 / 2}+\sqrt[4]{\tilde{\rho}_{\mathcal{M}}\left(y^{1}, y^{2}\right)}\right. \\
\left.+\sqrt[4]{\tilde{\rho}_{\mathcal{N}}\left(z^{1}, z^{2}\right)}\right\}+\varepsilon
\end{array}
$$

Hence $\forall \varepsilon>0, \exists \delta^{\prime}=\delta^{\prime}\left(\varepsilon, K, C, D, T, \mid g \|_{\infty}\right)>0$ such that $\forall x^{1}, x^{2} \in D, y^{1}, y^{2} \in$ $\mathcal{M}, z^{1}, z^{2} \in \mathcal{N},\left|x^{1}-x^{2}\right|+\tilde{\rho}_{\mathcal{M}}\left(y^{1}, y^{2}\right)+\tilde{\rho}_{\mathcal{N}}\left(z^{1}, z^{2}\right)<\delta^{\prime} \Rightarrow \mid J\left(t, x^{1}, y^{1}, z^{1}\right)-$ $J\left(t, x^{2}, y^{2}, z^{2}\right) \mid<2 \varepsilon$. That is, for $t \in[0, T], J(t, x, y, z, g)$ is uniformly continuous 
on $D \times \mathcal{M} \times \mathcal{N}$. The proof that $J$ is continuous in $t$ is easier. For fixed $t, s \geq 0, x \in$ $D, y \in \mathcal{M}, z \in \mathcal{N}$

$$
\begin{aligned}
& |J(t, x, y, z)-J(s, x, y, z)| \\
& \quad \leq \mathbf{E}_{x}^{y, z}\left|\int_{s \wedge \tau}^{t \wedge \tau} f\left(y_{r}, z_{r}, x_{r}\right) e^{-\varphi_{r}} d r\right|+\mathbf{E}_{x}^{y, z}\left|g\left(x_{t \wedge \tau}\right) e^{-\varphi_{t \wedge \tau}}-g\left(x_{s \wedge \tau}\right) e^{-\varphi_{s \wedge \tau}}\right| \\
& \leq\|f\|_{\infty} \mathbf{E}_{x}^{y, z}|t \wedge \tau-s \wedge \tau|+\mathbf{E}_{x}^{y, z}\left|g\left(x_{t \wedge \tau}\right)\right| \mid e^{-\varphi_{t \wedge \tau}}-e^{-\varphi_{s \wedge \tau} \mid} \\
& \quad+\mathbf{E}_{x}^{y, z}\left|g\left(x_{t \wedge \tau}\right)-g\left(x_{s \wedge \tau}\right)\right| \\
& \leq\|f\|_{\infty}|t-s|+\|g\|_{\infty} \mathbf{E}_{x}^{y, z}\left|\varphi_{s \wedge \tau}-\varphi_{t \wedge \tau}\right|+\mathbf{E}_{x}^{y, z}\left|g\left(x_{t \wedge \tau}\right)-g\left(x_{s \wedge \tau}\right)\right| \\
& \leq|t-s|\left(\|f\|_{\infty}+C\|g\|_{\infty}\right)+\mathbf{E}_{x}^{y, z}\left|g\left(x_{t \wedge \tau}\right)-g\left(x_{s \wedge \tau}\right)\right|, \\
& \mathbf{E}_{x}^{y, z}\left|x_{t \wedge \tau}-x_{s \wedge \tau}\right|^{2} \leq 2\left\{\mathbf{E}_{x}^{y, z}\left|\int_{s \wedge \tau}^{t \wedge \tau}\left\|\sigma\left(y_{r}, z_{r}, x_{r}\right)\right\|^{2} d r\right|\right. \\
& \left.\quad+\mathbf{E}_{x}^{y, z}\left|\int_{s \wedge \tau}^{t \wedge \tau}\right| b\left(y_{r}, z_{r}, x_{r}\right)|d r|^{2}\right\} \\
& \quad \leq 2 C^{2}\left\{|t-s|+|t-s|^{2}\right\} .
\end{aligned}
$$

Since $g$ is uniformly continuous in $D, \forall \varepsilon>0, \exists \delta(\varepsilon)>0$ such that $\forall \xi, \xi^{\prime} \in D$, $\left|\xi-\xi^{\prime}\right|<\delta(\varepsilon)$ implies $\left|g(\xi)-g\left(\xi^{\prime}\right)\right|<\varepsilon$. Hence by Chebyshev's inequality,

$$
\begin{aligned}
\mathbf{E}_{x}^{y, z}\left|g\left(x_{t \wedge \tau}\right)-g\left(x_{s \wedge \tau}\right)\right| & \leq \varepsilon+2\|g\|_{\infty} \mathbf{P}_{x}^{y, z}\left\{\left|x_{t \wedge \tau}-x_{s \wedge \tau}\right| \geq \delta\right\} \\
& \leq \varepsilon+\frac{4 C^{2}\|g\|_{\infty}}{\delta^{2}}\left\{|t-s|+|t-s|^{2}\right\} .
\end{aligned}
$$

Thus, for any $(x, y, z) \in D \times \mathcal{M} \times \mathcal{N}$,

$$
\begin{aligned}
|J(t, x, y, z)-J(s, x, y, z)| \leq & |t-s|\left(\|f\|_{\infty}+C\|g\|_{\infty}\right) \\
& +\frac{4 C^{2}\|g\|_{\infty}}{\delta^{2}}\left\{|t-s|+|t-s|^{2}\right\}+\varepsilon .
\end{aligned}
$$

Thus $\forall \varepsilon>0, \exists \delta^{\prime}=\delta^{\prime}\left(\varepsilon, C,\|g\|_{\infty}\right)$, such that $|t-s|<\delta^{\prime}$ implies $\mid \sup _{x, y, z} J(t, x, y, z)-$ $\sup _{x, y, z} J(s, x, y, z) \mid<2 \varepsilon$. Thus $J$ is (uniformly) continuous in $t$, uniformly with respect $x, y, z$

to $x, y, z$.

The fact that $J$ and the related functional (1.5) below are uniformly bounded, independent of $T$, follows from Assumption 1.0 and Itô's formula, since for every $x \in D, y \in \mathcal{M}, z \in \mathcal{N}$ and $t>0$,

$$
0 \leq \mathbf{E}_{x}^{y, z} \psi\left(x_{\tau \wedge t}\right)=\psi(x)+\mathbf{E}_{x}^{y, z} \int_{0}^{\tau \wedge t} L^{0}\left(y_{r}, z_{r}, x_{r}\right) \psi\left(x_{r}\right) d r \leq \psi(x)-\mathbf{E}_{x}^{y, z}[\tau \wedge t] .
$$

Hence by the monotone convergence theorem, $\sup _{y, z, x} \mathbf{E} \tau^{y, z, x} \leq \max _{D} \psi(x):=$ $|\psi|_{0, D}$. This inequality, our assumptions on $f$, and the elementary estimate

$$
\left|\sup _{y} \inf _{z} J\left(t, x^{1}, y, z\right)-\sup _{y} \inf _{z} J\left(t, x^{2}, y, z\right)\right| \leq \sup _{y} \sup _{z}\left|J\left(t, x^{1}, y, z\right)-J\left(t, x^{2}, y, z\right)\right|
$$


immediately imply

Corollary 1.3. For $g \in C(\bar{D})$ and $t \in[0, T]$, for $\hat{\mathcal{M}} \subset \mathcal{M}, \hat{\mathcal{N}} \subset \mathcal{N}$, the function

$$
J(t) g(x):=\sup _{y \in \hat{\mathcal{M}}} \inf _{z \in \hat{\mathcal{N}}} \mathbf{E}_{x}^{y, z}\left[\int_{0}^{t \wedge \tau} f\left(y_{r}, z_{r}, x_{r}\right) e^{-\varphi_{r}} d r+g\left(x_{t \wedge \tau}\right) e^{-\varphi_{t \wedge \tau}}\right]
$$

belongs to $C_{b}(D)$.

Remark. Since our continuity (in $x$ ) estimates rely entirely on continuity estimates for $J(t, x, y, z)$ which are uniform in $y, z$, our corollary holds for functionals $J(t) g$ in which inf and sup are reversed. This statement holds as well for the next few theorems. By our mean exit time estimate, $\forall x \in D,|J(t) g(x)| \leq N\|f\|_{\infty}+\|g\|_{\infty}$, where $N$ is independent of $t$. Moreover, it follows from the proof of Theorem 1.2 that $g \in C_{b}^{0, \beta}(D)$ implies $J(t) g \in C_{b}^{0, \beta / 2}(D), \beta \in(0,1]$. Furthermore, our previous estimate (1.2), obtained for $t \in[0, T]$, can be obtained for $\forall t \geq 0$, simply by redefining our metric on $\mathcal{M}, \mathcal{N}$ to be independent of $T$; e.g., instead of $\tilde{\rho}_{\mathcal{M}}$, define $\rho_{\mathcal{M}}^{*}\left(y^{1}, y^{2}\right):=\mathbf{E} \int_{0}^{\infty} \rho_{Y}\left(y_{r}^{1}, y_{r}^{2}\right) e^{-r} d r$. It is clear then that (1.2) (with $\rho^{*}$ in place of $\rho$ and a different $C_{2}$ ) will hold for all $t \geq 0$.

Theorem 1.4. For $x \in D$ and $g \in C(\bar{D})$, define

$$
v(x)=\sup _{y \in \hat{\mathcal{M}}} \inf _{z \in \hat{\mathcal{N}}} \mathbf{E}_{x}^{y, z}\left[\int_{0}^{\tau} f\left(y_{r}, z_{r}, x_{r}\right) e^{-\varphi_{r}} d r+g\left(x_{\tau}\right) e^{-\varphi_{\tau}}\right] .
$$

Then for $J$ in (1.5), we have

$$
\lim _{t \rightarrow \infty} J(t) g(x)=v(x)
$$

Proof. Recall that $\psi \geq 0$ in $D$. Setting $\mu=\frac{1}{2|\psi|_{\infty}}$, Itô's formula gives

$$
\begin{aligned}
0=\mathbf{E}_{x}^{y, z} \psi\left(x_{\tau}\right) e^{\mu \tau} & =\psi(x)+\mathbf{E}_{x}^{y, z} \int_{0}^{\tau}\left[L^{0}\left(y_{r}, z_{r}, x_{r}\right) \psi\left(x_{r}\right)+\mu \psi\left(x_{r}\right)\right] e^{\mu r} d r \\
& \leq \psi(x)-\frac{1}{2} \mathbf{E}_{x}^{y, z} \int_{0}^{\tau} e^{\mu r} d r
\end{aligned}
$$

from which it follows that $\mathbf{E}_{x}^{y, z} e^{\mu \tau} \leq 2$. Hence for $x \in D$,

$$
\begin{aligned}
|J(t) g(x)-v(x)| \leq \sup _{y \in \mathcal{M}} \sup _{z \in \mathcal{N}}\left\{\mathbf{E}_{x}^{y, z}\right. & \int_{t \wedge \tau}^{\tau}\left|f\left(y_{r}, z_{r}, x_{r}\right)\right| e^{-\varphi_{r}} d r \\
& \left.+\mathbf{E}_{x}^{y, z}\left|g\left(x_{t \wedge \tau}\right) e^{-\varphi_{t \wedge \tau}}-g\left(x_{\tau}\right) e^{-\varphi_{\tau}}\right|\right\} .
\end{aligned}
$$

But

$$
\begin{aligned}
\mathbf{E}_{x}^{y, z} \int_{t \wedge \tau}^{\tau}\left|f\left(y_{r}, z_{r}, x_{r}\right)\right| e^{-\varphi_{r}} d r & \leq\|f\|_{\infty} \mathbf{E}_{x}^{y, z}\left[(\tau-t) I_{\tau>t}\right] \\
& \leq C \sqrt{\mathbf{E}_{x}^{y, z} \tau^{2}} \sqrt{\mathbf{P}_{x}^{y, z}\{\tau>t\}} \leq \frac{C 4}{\mu} e^{-\frac{\mu t}{2}}
\end{aligned}
$$


By the Mean Value Theorem, $e^{-\varphi_{t \wedge \tau}}-e^{-\varphi_{\tau}} \leq \varphi_{\tau}-\varphi_{t \wedge \tau}=\int_{t \wedge \tau}^{\tau} c\left(y_{r}, z_{r}, x_{r}\right) d r \leq$ $C(\tau-t \wedge \tau)$ and hence

$$
\begin{aligned}
\mathbf{E}_{x}^{y, z}\left|g\left(x_{t \wedge \tau}\right) e^{-\varphi_{t \wedge \tau}}-g\left(x_{\tau}\right) e^{-\varphi_{\tau}}\right| \leq & \|g\|_{\infty} \mathbf{E}_{x}^{y, z}\left(e^{-\varphi_{t \wedge \tau}}-e^{-\varphi_{\tau}}\right) \\
& +\mathbf{E}_{x}^{y, z}\left|g\left(x_{t \wedge \tau}\right)-g\left(x_{\tau}\right)\right| \\
\leq & \|g\|_{\infty} C \mathbf{E}_{x}^{y, z}\left[(\tau-t) I_{\tau>t}\right]+2\|g\|_{\infty} \mathbf{P}_{x}^{y, z}\{\tau>t\} \\
\leq & 4\|g\|_{\infty} e^{-\frac{\mu t}{2}}\left(\frac{C}{\mu}+1\right) .
\end{aligned}
$$

Putting all this together yields

$$
|J(t) g(x)-v(x)| \leq 4 e^{-\frac{\mu t}{2}}\left\{\frac{C}{\mu}+\|g\|_{\infty}\left(\frac{C}{\mu}+1\right)\right\} .
$$

Remark. By the proof of our previous theorem, we know that for $g \in C_{b}(D), J(t) g$ is uniformly continuous in $t$ on $[0, \infty)$. If we knew $J(t)$ were a semigroup on some subset of $C_{b}(D)$, then our function $v(x)=\lim _{t \rightarrow \infty} J(t) g(x)$ would satisfy a dynamic programming principle, provided $v \in C_{b}(D)$. Indeed, in this case, for any $t \geq 0$,

$$
\begin{aligned}
v(x) & =\lim _{s \rightarrow \infty} J(t+s) g(x)=\lim _{s \rightarrow \infty} J(t)[J(s) g(x)] \\
& =J(t)\left[\lim _{s \rightarrow \infty} J(s) g(x)\right]=J(s) v(x) \\
& =\sup _{y \in \hat{\mathcal{M}}} \inf _{z \in \hat{\mathcal{N}}} \mathbf{E}_{x}^{y, z}\left[\int_{0}^{t \wedge \tau} f\left(x_{r}, y_{r}, z_{r}\right) e^{-\varphi_{r}} d r+v\left(x_{t \wedge \tau}\right) e^{-\varphi_{t \wedge \tau}}\right] .
\end{aligned}
$$

\section{Continuity PROPERTies of VAlue-type Functions}

By functions of value-type, we mean functions of the general form (1.6) (and with inf/sup reversed). The continuity results in this section hold, in particular, for the probabilistic solutions $(0.7),(0.8)$ of the Isaacs equations to be introduced in $\S 3$, which we call the value functions. From the theory of pde it is known that in the nondegenerate case, continuous viscosity solutions of the Dirichlet problem for Isaacs equations with bounded, Lipschitz coefficients (arbitrary continuous boundary values) are locally $C^{1, \alpha}(D)$. In the degenerate case, with positive discount factor, viscosity solutions are unique (see theorem II.2 in [IL) and, in the case of a global barrier, locally Lipschitz in $D$. We prove the Lipschitz continuity of functions of value-type (see Theorem 2.3) under the additional assumption that $g \in C^{2}(D)$.

First, we give a few preliminaries. Letting $T \rightarrow \infty$ in the proof of Lemma 1.1 and using the fact that $\sup _{y, z, x} \mathbf{E} \tau^{y, z, x} \leq|\psi|_{0, D}$ yields

$$
\mathbf{E}^{y, z}\left|\tau^{1}-\tau^{2}\right| \leq K \cdot \mathbf{E}^{y, z}\left|x_{\tau}^{1}-x_{\tau}^{2}\right|,
$$

where $\tau=\tau^{1} \wedge \tau^{2}$. Recalling that $\exists \mu=\mu(\psi)>0$ for which $\sup _{y, z, x} \mathbf{E}_{x}^{y, z} e^{\mu \tau} \leq 2$, $\forall t>0$, we have

$$
\begin{aligned}
\mathbf{E}^{y, z}\left|x_{\tau}^{1}-x_{\tau}^{2}\right| & =\mathbf{E}^{y, z}\left|x_{\tau}^{1}-x_{\tau}^{2}\right| I_{\tau \leq t}+\mathbf{E}^{y, z}\left|x_{\tau}^{1}-x_{\tau}^{2}\right| I_{\tau>t} \\
& \leq \mathbf{E}^{y, z} \sup _{0 \leq \leq \leq t}\left|x_{s}^{1}-x_{s}^{2}\right|+\operatorname{diam}(D) \cdot \mathbf{P}^{y, z}\left\{\tau^{1}>t\right\} \\
& \leq C e^{C t}\left|x^{1}-x^{2}\right|+2 \operatorname{diam}(D) e^{-\mu t} .
\end{aligned}
$$

The right-hand side is of the form $A e^{C t}+B e^{-\mu t}$, which for small $\left|x^{1}-x^{2}\right|$ attains its absolute minimum over $(0, \infty)$, at the point $t=t_{0}$ satisfying $A C e^{C t_{0}}=B \mu e^{-\mu t_{0}}$. This immediately implies

$$
\mathbf{E}^{y, z}\left|x_{\tau}^{1}-x_{\tau}^{2}\right| \leq N \cdot\left|x^{1}-x^{2}\right|^{\alpha},
$$


and hence

$$
\mathbf{E}^{y, z}\left|\tau^{1}-\tau^{2}\right| \leq K N \cdot\left|x^{1}-x^{2}\right|^{\alpha},
$$

where $\alpha=\frac{\mu}{C+\mu}$ and $N=N(C, \mu, \operatorname{diam}(D))$. Estimates of $\mathbf{E}^{y, z} \tau \int_{0}^{\tau}\left|x_{r}^{1}-x_{r}^{2}\right| d r$ and $\mathbf{E}^{y, z} \int_{0}^{\tau}\left|x_{r}^{1}-x_{r}^{2}\right| d r$ are handled the same way. For example, for any $t>0$,

$$
\begin{aligned}
\mathbf{E}^{y, z} \tau \int_{0}^{\tau}\left|x_{r}^{1}-x_{r}^{2}\right| d r= & \mathbf{E}^{y, z}\left[\tau \int_{0}^{\tau}\left|x_{r}^{1}-x_{r}^{2}\right| d r \cdot I_{\tau \leq t}\right] \\
& +\mathbf{E}^{y, z}\left[\tau \int_{0}^{\tau}\left|x_{r}^{1}-x_{r}^{2}\right| d r \cdot I_{\tau>t}\right] \\
\leq & t \int_{0}^{t} \mathbf{E}^{y, z}\left|x_{r}^{1}-x_{r}^{2}\right| d r+\operatorname{diam}(D) \mathbf{E}^{y, z}\left[\tau^{2} \cdot I_{\tau>t}\right] \\
\leq & t \sqrt{C_{2}}\left|x^{1}-x^{2}\right| \int_{0}^{t} e^{C_{2} r} d r \\
& +\operatorname{diam}(D) \sqrt{\mathbf{E}^{y, z} \tau^{4}} \cdot \sqrt{\mathbf{P}^{y, z}\{\tau>t\}} \\
\leq & \left|x^{1}-x^{2}\right| \frac{e^{\left(1+C_{2}\right) t}}{\sqrt{C_{2}}}+\operatorname{diam}(D) \frac{7}{\mu^{2}} e^{-\mu t / 2}
\end{aligned}
$$

and taking the minimum over $t \in(0, \infty)$ gives for $\beta=\frac{\mu}{2\left(1+C_{2}\right)+\mu}$ and $N=$ $N(C, \mu, \operatorname{diam}(D))$,

$$
\mathbf{E}^{y, z} \tau \int_{0}^{\tau}\left|x_{r}^{1}-x_{r}^{2}\right| d r \leq N \cdot\left|x^{1}-x^{2}\right|^{\beta}
$$

We use these estimates to establish continuity properties of value-type functions. For $g \in C(\bar{D}), y \in \mathcal{M}, z \in \mathcal{N}, x \in D$, we define

$$
\begin{aligned}
J(y, z, x, g):=\mathbf{E}_{x}^{y, z}\left[\int_{0}^{\tau} f\left(y_{r}, z_{r}, x_{r}\right) e^{-\varphi_{r}} d r+g\left(x_{\tau}\right) e^{-\varphi_{\tau}}\right] \\
\text { where } \varphi_{t}^{y, z, x}=\int_{0}^{t} c\left(y_{s}, z_{s}, x_{s}^{y, z, x}\right) d s,
\end{aligned}
$$

$\tau^{y, z, x}=\tau_{D}^{y, z, x}:=\inf \left\{t \geq 0: x_{t}^{y, z, x} \notin D\right\}$, and $x_{t}^{y, z, x}$ is the solution of our stochastic integral equation.

Theorem 2.1. Let $g \in C(\bar{D})$, and let $\hat{\mathcal{M}} \subset \mathcal{M}, \hat{\mathcal{N}} \subset \mathcal{N}$. Then the function

$$
v(x):=\sup _{y \in \hat{\mathcal{M}}} \inf _{z \in \hat{\mathcal{N}}} J(y, z, x, g)
$$

belongs to $C(\bar{D})$.

Proof. We will show that (i) $v \in C_{b}(D)$ and (ii) $\forall x_{0} \in \partial D, \lim _{x \in D, x \rightarrow x_{0}} v(x)=g\left(x_{0}\right)$. The fact that $v$ is bounded follows from the uniform boundedness of $f, g$ and the estimate $\sup _{y, z, x} \mathbf{E} \tau^{y, z, x} \leq|\psi|_{0, D}$. To prove (i), fix $x^{1}, x^{2} \in D$. Omitting the obvious dependence on $g$, we have

$$
\begin{aligned}
\left|v\left(x^{1}\right)-v\left(x^{2}\right)\right| & =\left|\sup _{y \in \hat{\mathcal{M}}} \inf _{z \in \hat{\mathcal{N}}} J\left(y, z, x^{1}\right)-\sup _{y \in \hat{\mathcal{M}}} \inf _{z \in \hat{\mathcal{N}}} J\left(y, z, x^{2}\right)\right| \\
& \leq \sup _{y \in \mathcal{M}} \sup _{z \in \mathcal{N}}\left|J\left(y, z, x^{1}\right)-J\left(y, z, x^{2}\right)\right| .
\end{aligned}
$$


For fixed $y \in \mathcal{M}, z \in \mathcal{N}$ and $x^{1}, x^{2} \in D$, set $\tau=\tau^{y, z, x^{1}} \wedge \tau^{y, z, x^{2}}=\tau^{1} \wedge \tau^{2}$. Then

$$
\begin{aligned}
J\left(y, z, x^{1}\right)-J\left(y, z, x^{2}\right)= & \mathbf{E}^{y, z}\left[\int_{0}^{\tau^{1}} f\left(y_{r}, z_{r}, x_{r}^{1}\right) e^{-\varphi_{r}^{1}} d r-\int_{0}^{\tau^{2}} f\left(y_{r}, z_{r}, x_{r}^{2}\right) e^{-\varphi_{r}^{2}} d r\right] \\
& +\mathbf{E}^{y, z}\left[g\left(x_{\tau^{1}}^{1}\right) e^{-\varphi_{\tau^{1}}^{1}}-g\left(x_{\tau^{2}}^{2}\right) e^{-\varphi_{\tau^{2}}^{2}}\right] \\
:= & T_{1}+T_{2} .
\end{aligned}
$$

Writing the expression inside the expectation of $T_{1}$ (in obvious abbreviated notation) as

$$
\int_{\tau}^{\tau^{1}} f_{r}^{1} e^{-\varphi_{r}^{1}} d r+\int_{0}^{\tau} f_{r}^{1}\left[e^{-\varphi_{r}^{1}}-e^{-\varphi_{r}^{2}}\right] d r+\int_{0}^{\tau} e^{-\varphi_{r}^{2}}\left[f_{r}^{1}-f_{r}^{2}\right] d r+\int_{\tau^{2}}^{\tau} f_{r}^{2} e^{-\varphi_{r}^{2}} d r
$$

by our previous estimates, we have

$$
\begin{aligned}
\left|T_{1}\right| \leq & C \mathbf{E}^{y, z}\left(\tau^{1}-\tau\right)+C^{2} \mathbf{E}^{y, z} \int_{0}^{\tau} \int_{0}^{r}\left|x_{s}^{1}-x_{s}^{2}\right| d s d r \\
& +C \mathbf{E}^{y, z} \int_{0}^{\tau}\left|x_{r}^{1}-x_{r}^{2}\right| d r+C \mathbf{E}^{y, z}\left(\tau^{2}-\tau\right) \\
\leq & 2 C \mathbf{E}^{y, z}\left|\tau^{1}-\tau^{2}\right|+C^{2} \mathbf{E}^{y, z} \tau \int_{0}^{\tau}\left|x_{s}^{1}-x_{s}^{2}\right| d s+C \mathbf{E}^{y, z} \int_{0}^{\tau}\left|x_{r}^{1}-x_{r}^{2}\right| d r .
\end{aligned}
$$

As usual, by the uniform continuity of $g$ in $\bar{D}, \forall \varepsilon>0, \exists \delta(\varepsilon)>0$ with

$$
\begin{aligned}
\left|T_{2}\right| \leq & \|g\|_{\infty} \mathbf{E}^{y, z}\left|\varphi_{\tau^{1}}^{1}-\varphi_{\tau^{2}}^{2}\right|+\mathbf{E}^{y, z}\left|g\left(x_{\tau^{1}}^{1}\right)-g\left(x_{\tau^{2}}^{2}\right)\right| \\
\leq & \|g\|_{\infty} 2 C\left\{\mathbf{E}^{y, z}\left|\tau^{1}-\tau^{2}\right|+\mathbf{E}^{y, z} \int_{0}^{\tau}\left|x_{r}^{1}-x_{r}^{2}\right| d r\right\} \\
& +2\|g\|_{\infty} \mathbf{P}^{y, z}\left\{\left|x_{\tau^{1}}^{1}-x_{\tau^{2}}^{2}\right|>\delta\right\}+\varepsilon .
\end{aligned}
$$

By Chebyshev's inequality,

$$
\mathbf{P}^{y, z}\left\{\left|x_{\tau^{1}}^{1}-x_{\tau^{2}}^{2}\right|>\delta\right\} \leq \frac{3}{\delta}\left\{\mathbf{E}^{y, z}\left|x_{\tau^{1}}^{1}-x_{\tau}^{1}\right|+\mathbf{E}^{y, z}\left|x_{\tau}^{1}-x_{\tau}^{2}\right|+\mathbf{E}^{y, z}\left|x_{\tau}^{2}-x_{\tau^{2}}^{2}\right|\right\} .
$$

From

we have

$$
\left|x_{\tau^{1}}^{1}-x_{\tau}^{1}\right| \leq\left|\int_{\tau}^{\tau^{1}} \sigma\left(y_{r}, z_{r}, x_{r}^{1}\right) d w_{r}\right|+\left|\int_{\tau}^{\tau^{1}} b\left(y_{r}, z_{r}, x_{r}^{1}\right) d r\right|,
$$

$$
\begin{aligned}
\mathbf{E}^{y, z}\left|x_{\tau^{1}}^{1}-x_{\tau}^{1}\right| & \leq N C \mathbf{E}^{y, z}\left(\tau^{1}-\tau\right)^{1 / 2}+C \mathbf{E}^{y, z}\left(\tau^{1}-\tau\right) \\
& \leq N_{1}(C)\left\{\mathbf{E}^{y, z}\left|\tau^{1}-\tau^{2}\right|^{1 / 2}+\mathbf{E}^{y, z}\left|\tau^{1}-\tau^{2}\right|\right\} \\
& \leq N_{1}(C)\left\{\left(\mathbf{E}^{y, z}\left|\tau^{1}-\tau^{2}\right|\right)^{1 / 2}+\mathbf{E}^{y, z}\left|\tau^{1}-\tau^{2}\right|\right\}
\end{aligned}
$$

and hence

$$
\begin{aligned}
\left|T_{2}\right| \leq C_{2}\|g\|_{\infty}\left\{\mathbf{E}^{y, z}\left|\tau^{1}-\tau^{2}\right|+\mathbf{E}^{y, z} \int_{0}^{\tau} \mid\right. & x_{r}^{1}-x_{r}^{2} \mid d r+\frac{N_{3}(C)}{\delta}\left(\left(\mathbf{E}^{y, z}\left|\tau^{1}-\tau^{2}\right|\right)^{1 / 2}\right. \\
& \left.\left.+\mathbf{E}^{y, z}\left|\tau^{1}-\tau^{2}\right|+\mathbf{E}^{y, z}\left|x_{\tau}^{1}-x_{\tau}^{2}\right|\right)\right\}+\varepsilon
\end{aligned}
$$

Estimates (2.1)-(2.3) and routine calculations give

$$
\left|T_{1}+T_{2}\right| \leq C_{4}\left|x^{1}-x^{2}\right|^{\alpha}+C_{2}\|g\|_{\infty}\left\{\left|x^{1}-x^{2}\right|^{\gamma_{1}}+\frac{N_{4}}{\delta}\left|x^{1}-x^{2}\right|^{\gamma_{2}}\right\}+\varepsilon,
$$


where $\alpha, \gamma_{1}, \gamma_{2}$ depend only on $C, \mu, D$ with the constants depending additionally on $K$. From this it follows that $\forall \varepsilon>0, \exists \delta^{\prime}=\delta^{\prime}\left(\varepsilon, C, D, \mu, K,\|g\|_{\infty}\right)$ such that $\forall x^{1}, x^{2} \in D$, if $\left|x^{1}-x^{2}\right|<\delta^{\prime}$, then

$$
\left|v\left(x^{1}\right)-v\left(x^{2}\right)\right| \leq \sup _{y \in \mathcal{M}} \sup _{z \in \mathcal{N}}\left|J\left(y, z, x^{1}\right)-J\left(y, z, x^{2}\right)\right|<2 \varepsilon,
$$

which proves (i). To prove (ii), observe that

$|v(x)-g(x)| \leq \sup _{y \in \mathcal{M}} \sup _{z \in \mathcal{N}}\left\{\mathbf{E}_{x}^{y, z}\left|\int_{0}^{\tau} f\left(y_{r}, z_{r}, x_{r}\right) e^{-\varphi_{r}} d r\right|+\mathbf{E}_{x}^{y, z}\left|g\left(x_{\tau}\right) e^{-\varphi_{\tau}}-g(x)\right|\right\}$.

Applying Itô's theorem to the barrier function $\psi$ gives $\sup _{y, z} \mathbf{E}_{x}^{y, z} \tau \leq \psi(x)$. By the uniform continuity of $g$ in $\bar{D}$, and then Chebyshev's inequality, we have $\forall \varepsilon>$ $0, \exists \delta(\varepsilon)>0$ with

$$
\begin{aligned}
\mathbf{E}_{x}^{y, z}\left|g\left(x_{\tau}\right) e^{-\varphi_{\tau}}-g(x)\right| & \leq\|g\|_{\infty} \mathbf{E}_{x}^{y, z}\left(1-e^{-\varphi_{\tau}}\right)+\mathbf{E}_{x}^{y, z}\left|g\left(x_{\tau}\right)-g(x)\right| \\
& \leq\|g\|_{\infty} C \mathbf{E}_{x}^{y, z} \tau+\frac{2\|g\|_{\infty}}{\delta} \mathbf{E}_{x}^{y, z}\left|x_{\tau}-x\right|+\varepsilon
\end{aligned}
$$

This, $\|f\|_{\infty} \leq C$ and the inequalities

$$
\mathbf{E}_{x}^{y, z}\left|x_{\tau}-x\right| \leq C\left\{\left(\mathbf{E}_{x}^{y, z} \tau\right)^{1 / 2}+\mathbf{E}_{x}^{y, z} \tau\right\} \leq C\{\sqrt{\psi(x)}+\psi(x)\}
$$

yield, for $x \in D$ and any $\varepsilon>0$,

$$
|v(x)-g(x)| \leq C \psi(x)\left(1+\|g\|_{\infty}\right)+\frac{2 C\|g\|_{\infty}}{\delta(\varepsilon)}\{\sqrt{\psi(x)}+\psi(x)\}+\varepsilon .
$$

From this, the inequality $\left|v(x)-g\left(x_{0}\right)\right| \leq|v(x)-g(x)|+\left|g(x)-g\left(x_{0}\right)\right|$, and the fact that $\lim _{x \rightarrow x_{0}} \psi(x)=0$, it follows that $\forall \varepsilon>0, \exists \delta^{\prime}=\delta^{\prime}\left(\varepsilon, C,\|g\|_{\infty}\right)$ such that $\forall x \in D$, if $\left|x-x_{0}\right|<\delta^{\prime}$, then $\left|v(x)-g\left(x_{0}\right)\right|<3 \varepsilon$, proving (ii).

Remark. Using the same argument as in (ii), we can show that for $x_{0} \in \partial D$, $\lim _{x \in D, x \rightarrow x_{0}} J(t) g(x)=g\left(x_{0}\right)$. This, along with Corollary 1.3, implies that for $g \in$ $C(\bar{D}), J(t) g \in C(\bar{D})$. Using the same techniques as in (i), we can show that if $g \in C^{0, \beta}(\bar{D}), \beta \in(0,1]$, then there is a constant $N=N(d, \mu, C, K, D, \beta)$ and $\gamma=\gamma(d, \mu, C, D, \beta)$ such that for any $x^{1}, x^{2} \in D$,

$$
\left|v\left(x^{1}\right)-v\left(x^{2}\right)\right| \leq N\left(1+\|g\|_{0, \beta}\right) \cdot\left|x^{1}-x^{2}\right|^{\gamma}
$$

We now prove the Lipschitz continuity of the value-type functions, under the additional assumptions that (i) $g \in C^{2}(D)$ and (ii) the lower bound of the discount factor is large compared to the Lipschitz constant for coefficients $\sigma$ and $b$ (assumption (2.4) below). Our techniques are similar to those found in Theorems 2.3 in both [L] and LM1. More precisely, assumption (ii) is that

$$
\begin{aligned}
& \inf _{\substack{x \in E_{d} \\
(y, z) \in Y \times Z}} c(y, z, x)=c_{0}>\mu_{0} \text {, where } \\
\mu_{0}= & \sup _{\substack{x, x^{\prime} \in E_{d} \\
(y, z) \in Y \times Z}}\left\{\frac{\left\|\sigma(y, z, x)-\sigma\left(y, z, x^{\prime}\right)\right\|^{2}}{2\left|x-x^{\prime}\right|^{2}}+\frac{\left(b(y, z, x)-b\left(y, z, x^{\prime}\right)\right) \cdot\left(x-x^{\prime}\right)}{\left|x-x^{\prime}\right|^{2}}\right\} .
\end{aligned}
$$

Lemma 2.2. For all admissible controls $y \in \mathcal{M}, z \in \mathcal{N}$ and any $\mathcal{F}_{t}$-Markov time $\theta$,

$$
\mathbf{E}^{y, z}\left[\left|x_{\theta}^{x}-x_{\theta}^{x^{\prime}}\right|^{2} e^{-2 \mu_{0} \theta}\right] \leq\left|x-x^{\prime}\right|^{2}
$$


Proof. Writing

$$
\begin{aligned}
x_{t}^{y, z, x}-x_{t}^{y, z, x^{\prime}}=x-x^{\prime}+ & \int_{0}^{t}\left[\sigma\left(y_{r}, z_{r}, x_{r}^{x}\right)-\sigma\left(y_{r}, z_{r}, x_{r}^{x^{\prime}}\right)\right] d w_{r} \\
& +\int_{0}^{t}\left[b\left(y_{r}, z_{r}, x_{r}^{x}\right)-b\left(y_{r}, z_{r}, x_{r}^{x^{\prime}}\right)\right] d r,
\end{aligned}
$$

the definition of $\mu_{0}$, and Itô's formula applied to the function $z \rightarrow|z|^{2}$ yields

$$
\begin{aligned}
& \mathbf{E}^{y, z}\left[\left|x_{T \wedge \theta}^{x}-x_{T \wedge \theta}^{x^{\prime}}\right|^{2} e^{-2 \mu_{0} T \wedge \theta}\right] \\
& =\left|x-x^{\prime}\right|^{2} \\
& \quad+\mathbf{E}^{y, z} \int_{0}^{T \wedge \theta}\left\{\operatorname{tr}\left[\left(\sigma\left(y_{r}, z_{r}, x_{r}^{x}\right)-\sigma\left(y_{r}, z_{r}, x_{r}^{x^{\prime}}\right)\right)\left(\sigma\left(y_{r}, z_{r}, x_{r}^{x}\right)-\sigma\left(y_{r}, z_{r}, x_{r}^{x^{\prime}}\right)\right)^{*}\right]\right. \\
& \left.\quad+2\left(b\left(y_{r}, z_{r}, x_{r}^{x}\right)-b\left(y_{r}, z_{r}, x_{r}^{x^{\prime}}\right)\right) \cdot\left(x_{r}^{x}-x_{r}^{x^{\prime}}\right)-2 \mu_{0}\left|x_{r}^{x}-x_{r}^{x^{\prime}}\right|^{2}\right\} e^{-2 \mu_{0} r} d r . \\
& \leq\left|x-x^{\prime}\right|^{2} .
\end{aligned}
$$

Theorem 2.3. Under the above assumptions, there is a constant

$$
N=N\left(C, c_{0}, \mu_{0},|g|_{2, D},\left|\psi_{x}\right|_{0, D}\right)
$$

such that for any $x^{1}, x^{2} \in D$,

$$
\left|v\left(x^{1}\right)-v\left(x^{2}\right)\right| \leq N\left|x^{1}-x^{2}\right| .
$$

Proof. For any $x^{1}, x^{2} \in D$, as before,

$$
\begin{aligned}
\left|v\left(x^{1}\right)-v\left(x^{2}\right)\right| & =\left|\sup _{y \in \hat{\mathcal{M}}} \inf _{z \in \hat{\mathcal{N}}} J\left(y, z, x^{1}\right)-\sup _{y \in \hat{\mathcal{M}}} \inf _{z \in \hat{\mathcal{N}}} J\left(y, z, x^{2}\right)\right| \\
& \leq \sup _{y \in \mathcal{M}} \sup _{z \in \mathcal{N}}\left|J\left(y, z, x^{1}\right)-J\left(y, z, x^{2}\right)\right| .
\end{aligned}
$$

For any $y \in \mathcal{M}, z \in \mathcal{N}$ and $x^{1}, x^{2} \in D$, using the same " 1,2 " notation for convenience,

$$
\begin{aligned}
J\left(y, z, x^{1}\right)-J\left(y, z, x^{2}\right)= & \mathbf{E}^{y, z}\left[\int_{0}^{\tau^{1}} f_{r}^{1} e^{-\varphi_{r}^{1}} d r-\int_{0}^{\tau^{2}} f_{r}^{2} e^{-\varphi_{r}^{2}} d r\right] \\
& +\mathbf{E}^{y, z}\left[g\left(x_{\tau^{1}}^{1}\right) e^{-\varphi_{\tau^{1}}^{1}}-g\left(x_{\tau^{2}}^{2}\right) e^{-\varphi_{\tau^{2}}^{2}}\right] \\
:= & T_{1}+T_{2} .
\end{aligned}
$$

As before, and using the fact that $c \geq c_{0}$,

$$
\begin{aligned}
\left|T_{1}\right| \leq & \int_{0}^{\infty} \mathbf{E}^{y, z} I_{r \leq \tau^{1}}\left|f_{r}^{1}\right|\left|e^{-\varphi_{r}^{1}}-e^{-\varphi_{r}^{2}}\right| d r \\
& +\int_{0}^{\infty} \mathbf{E}^{y, z} I_{r \leq \tau^{1}} e^{-\varphi_{r}^{2}}\left|f_{r}^{1}-f_{r}^{2}\right| d r+\mathbf{E}^{y, z}\left|\int_{\tau^{2}}^{\tau^{1}}\right| f_{r}^{2}\left|e^{-\varphi_{r}^{2}} d r\right| \\
\leq & C \int_{0}^{\infty} \mathbf{E}^{y, z}\left|e^{-\varphi_{r}^{1}}-e^{-\varphi_{r}^{2}}\right| d r+C \int_{0}^{\infty} e^{-c_{0} r} \mathbf{E}^{y, z}\left|x_{r}^{1}-x_{r}^{2}\right| d r \\
& +\frac{C}{c_{0}} \mathbf{E}^{y, z}\left|e^{-c_{0} \tau^{1}}-e^{-c_{0} \tau^{2}}\right| .
\end{aligned}
$$


By the Mean Value Theorem, $e^{-\varphi_{t}^{1}}-e^{-\varphi_{t}^{2}}=e^{-\xi}\left(\varphi_{t}^{2}-\varphi_{t}^{1}\right.$ ), with $\xi \geq c_{0} t$ (since $\left.c \geq c_{0}\right)$. Moreover, $\left|\varphi_{t}^{1}-\varphi_{t}^{2}\right| \leq C \int_{0}^{t}\left|x_{s}^{1}-x_{s}^{2}\right| d s$. Hence

$$
\mathbf{E}^{y, z}\left|e^{-\varphi_{t}^{1}}-e^{-\varphi_{t}^{2}}\right| \leq \mathbf{E}^{y, z} e^{-\xi}\left|\varphi_{t}^{1}-\varphi_{t}^{2}\right| \leq C e^{-c_{0} t} \int_{0}^{t} \mathbf{E}^{y, z}\left|x_{s}^{1}-x_{s}^{2}\right| d s .
$$

By Lemma $2.2, \mathbf{E}^{y, z}\left|x_{t}^{1}-x_{t}^{2}\right| \leq\left|x^{1}-x^{2}\right| e^{\mu_{0} t}$. Hence by (2.6),

$$
\mathbf{E}^{y, z}\left|e^{-\varphi_{t}^{1}}-e^{-\varphi_{t}^{2}}\right| \leq \frac{C}{\mu_{0}}\left|x^{1}-x^{2}\right| e^{\left(\mu_{0}-c_{0}\right) t},
$$

which by (2.5), immediately yields

$$
\begin{aligned}
\left|T_{1}\right| \leq & \frac{C^{2}\left|x^{1}-x^{2}\right|}{\mu_{0}} \int_{0}^{\infty} e^{\left(\mu_{0}-c_{0}\right) r} d r+C\left|x^{1}-x^{2}\right| \int_{0}^{\infty} e^{\left(\mu_{0}-c_{0}\right) r} d r \\
& +\frac{C}{c_{0}} \mathbf{E}^{y, z}\left|e^{-c_{0} \tau^{1}}-e^{-c_{0} \tau^{2}}\right| .
\end{aligned}
$$

By assumption (iii), Itô's formula implies $\forall x \in D, y \in \mathcal{M}, z \in \mathcal{N}$,

$$
\left.0=\mathbf{E}_{x}^{y, z} \psi_{(} x_{\tau}\right)=\psi(x)+\mathbf{E}_{x}^{y, z} \int_{0}^{\tau} L^{0}\left(y_{r}, z_{r}, x_{r}\right) \psi\left(x_{r}\right) d r \leq \psi(x)-\mathbf{E}_{x}^{y, z} \tau .
$$

Hence $\mathbf{E} \tau^{y, z, x} \leq|\psi|_{0, D}$. Itô's formula implies that the process

$$
\beta_{t}^{y, z, x}:=\psi\left(x_{t \wedge \tau}\right) e^{-c_{0}(t \wedge \tau)}+\int_{0}^{t \wedge \tau} e^{-c_{0} r} d r,
$$

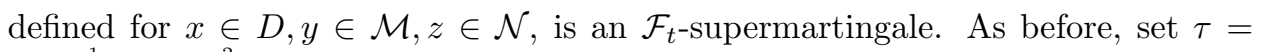
$\tau^{y, z, x^{1}} \wedge \tau^{y, z, x^{2}}=\tau^{1} \wedge \tau^{2}$. Since $\tau \leq \tau^{1}$, the definition of supermartingale implies

$$
\begin{aligned}
\frac{1}{c_{0}} \mathbf{E}^{y, z}\left[e^{-c_{0} \tau}-e^{-c_{0} \tau^{1}}\right] \leq & \mathbf{E}^{y, z}\left[\psi\left(x_{\tau}^{1}\right) e^{-c_{0} \tau}-\psi\left(x_{\tau^{1}}^{1}\right) e^{-c_{0} \tau^{1}}\right] \\
= & \mathbf{E}^{y, z}\left[\left\{\psi\left(x_{\tau}^{1}\right)-\psi\left(x_{\tau}^{2}\right)\right\} e^{-c_{0} \tau}\right] \\
& +\mathbf{E}^{y, z}\left[\psi\left(x_{\tau}^{2}\right) e^{-c_{0} \tau}-\psi\left(x_{\tau^{1}}^{1}\right) e^{-c_{0} \tau^{1}}\right] .
\end{aligned}
$$

Note that the quantity inside the expectation of our last summand is 0 a.s. on the set $\tau^{2} \leq \tau^{1}$, since $\psi \equiv 0$ on $\partial D$. By the Mean Value Theorem, $c_{0}>\mu_{0}$, and Lemma 2.2 ,

$$
\begin{aligned}
\frac{1}{c_{0}} \mathbf{E}^{y, z}\left[e^{-c_{0} \tau}-e^{-c_{0} \tau^{1}}\right] & \leq 2 \mathbf{E}^{y, z} e^{-c_{0} \tau}\left|\psi\left(x_{\tau}^{1}\right)-\psi\left(x_{\tau}^{2}\right)\right| \\
& \leq 2\left\|\psi_{x}\right\|_{\infty} \mathbf{E}^{y, z} e^{-c_{0} \tau}\left|x_{\tau}^{1}-x_{\tau}^{2}\right| \leq 2\left\|\psi_{x}\right\|_{\infty}\left|x^{1}-x^{2}\right|,
\end{aligned}
$$

and hence

which by (2.7) gives

$$
\mathbf{E}^{y, z}\left|e^{-c_{0} \tau^{1}}-e^{-c_{0} \tau^{2}}\right| \leq 4 c_{0}\left\|\psi_{x}\right\|_{\infty}\left|x^{1}-x^{2}\right|,
$$

$$
\left|T_{1}\right| \leq\left|x^{1}-x^{2}\right|\left\{\frac{C^{2}}{\mu_{0}\left(c_{0}-\mu_{0}\right)}+\frac{C}{c_{0}-\mu_{0}}+4 C\left\|\psi_{x}\right\|_{\infty}\right\} .
$$

To estimate $T_{2}$, we write

$$
\begin{aligned}
& \left|\mathbf{E}^{y, z}\left[g\left(x_{\tau^{1}}^{1}\right) e^{-\varphi_{\tau^{1}}^{1}}-g\left(x_{\tau^{2}}^{2}\right) e^{-\varphi_{\tau^{2}}^{2}}\right]\right| \\
& \leq\left|\mathbf{E}^{y, z}\left[g\left(x_{\tau^{1}}^{1}\right) e^{-\varphi_{\tau^{1}}^{1}}-g\left(x_{\tau}^{1}\right) e^{-\varphi_{\tau}^{1}}\right]\right|+\mathbf{E}^{y, z}\left|g\left(x_{\tau}^{1}\right) e^{-\varphi_{\tau}^{1}}-g\left(x_{\tau}^{2}\right) e^{-\varphi_{\tau}^{2}}\right| \\
& \quad+\left|\mathbf{E}^{y, z}\left[g\left(x_{\tau}^{2}\right) e^{-\varphi_{\tau}^{2}}-g\left(x_{\tau^{2}}^{2}\right) e^{-\varphi_{\tau^{2}}^{2}}\right]\right| .
\end{aligned}
$$


The first and third summands are estimated using Itô's formula

$$
\begin{aligned}
\left|\mathbf{E}^{y, z}\left[g\left(x_{\tau^{1}}^{1}\right) e^{-\varphi_{\tau^{1}}^{1}}-g\left(x_{\tau}^{1}\right) e^{-\varphi_{\tau}^{1}}\right]\right| & \leq \mathbf{E}^{y, z} \int_{\tau}^{\tau^{1}}\left|L\left(y_{r}, z_{r}, x_{r}\right) g\left(x_{r}^{1}\right)\right| e^{-\varphi_{r}^{1}} d r \\
& \leq N(C, d)\|g\|_{2, \infty} \mathbf{E}^{y, z} \int_{\tau}^{\tau^{1}} e^{-c_{0} r} d r \\
& \leq 2 N\|g\|_{2, \infty}\left\|\psi_{x}\right\|_{\infty}\left|x^{1}-x^{2}\right| .
\end{aligned}
$$

We handle the middle summand in the usual way:

$$
\left|g\left(x_{\tau}^{1}\right) e^{-\varphi_{\tau}^{1}}-g\left(x_{\tau}^{2}\right) e^{-\varphi_{\tau}^{2}}\right| \leq\|g\|_{\infty}\left|e^{-\varphi_{\tau}^{1}}-e^{-\varphi_{\tau}^{2}}\right|+e^{-c_{0} \tau}\left\|g_{x}\right\|_{\infty}\left|x_{\tau}^{1}-x_{\tau}^{2}\right| .
$$

As before, $\left|e^{-\varphi_{\tau}^{1}}-e^{-\varphi_{\tau}^{2}}\right| \leq C e^{-c_{0} \tau} \int_{0}^{\tau}\left|x_{r}^{1}-x_{r}^{2}\right| d r \leq C \int_{0}^{\infty} e^{-c_{0} r}\left|x_{r}^{1}-x_{r}^{2}\right| d r$. Taking the expectation in (2.9) and using Lemma 2.2, along with $c_{0}>\mu_{0}$, yields

$$
\mathbf{E}^{y, z}\left|g\left(x_{\tau}^{1}\right) e^{-\varphi_{\tau}^{1}}-g\left(x_{\tau}^{2}\right) e^{-\varphi_{\tau}^{2}}\right| \leq\left|x^{1}-x^{2}\right|\left\{\frac{\|g\|_{\infty} C}{c_{0}-\mu_{0}}+\left\|g_{x}\right\|_{\infty}\right\},
$$

and hence

$$
\left|T_{2}\right| \leq\left|x^{1}-x^{2}\right|\left\{4 C\|g\|_{2, \infty}\left\|\psi_{x}\right\|_{\infty}+\frac{\|g\|_{\infty} C}{c_{0}-\mu_{0}}+\left\|g_{x}\right\|_{\infty}\right\},
$$

which, along with (2.8) gives

$$
\left|v\left(x^{1}\right)-v\left(x^{2}\right)\right| \leq \sup _{y \in \mathcal{M}} \sup _{z \in \mathcal{N}}\left|J\left(y, z, x^{1}, g\right)-J\left(y, z, x^{2}, g\right)\right| \leq N\left|x^{1}-x^{2}\right| .
$$

\section{SEMigroup PROPERTIES}

In this section, we establish the dynamic programming principle for the upper and lower value functions $v^{+}(x), v^{-}(x)$ defined in (3.14), (3.15). We do this by showing that the upper and lower value functions $V^{+}(t) g, V^{-}(t) g$, defined in (3.4), form a semigroup on $C(\bar{D})$ (see the remark at the end of $\S 1$ ). Our setup is as follows: $(\Omega, \mathcal{F}, \mathbf{P})$ is a probablity space on which a $d$-dimensional Brownian motion $\left(w_{t}, \mathcal{F}_{t}\right)$ is defined. Here, for our filtration $\left\{\mathcal{F}_{t}\right\}_{t>0}$ of $\sigma$-algebras of $\Omega$, we take $\mathcal{F}_{t}:=\mathcal{F}_{t}^{w}=\sigma\left(w_{s}: 0 \leq s \leq t\right)$. We adapt the weak formulation of the differential game in which our probability space may vary, and hence admissible controls may be defined on another probability space (e.g. the canonical space). In the strong formulation of a differential game, the probability space is fixed. Consequently, controls may be defined only on the original space. The weak formulation has the obvious practical advantage of a wider class of admissible controls for the players. Even in the one-player setting, there are known examples in which optimal controls do not exist in the strong formulation, but do exist in the weak formulation.

Observe that by condition $(1.1)^{\prime}:$ if $\left(\Omega^{\prime}, \mathcal{G}, \mathbf{P}^{\prime}\right)$ is any probability space on which a $d$-dimensional Brownian motion $\left(\beta_{t}, \mathcal{G}_{t}\right)$ is defined, and if $y=y \cdot\left(\omega^{\prime}\right), z=z \cdot\left(\omega^{\prime}\right)$ are $\left\{\mathcal{G}_{t}\right\}$-progressively measurable processes with values in $Y$ and $Z$ respectively, then there exists a $\left(\mathbf{P}^{\prime}\right.$-a.s. $)$ unique, $\mathcal{G}_{t}$-measurable solution $x_{t}=x_{t}^{y, z, x}$ to the equation (defined on $\left(\Omega^{\prime}, \mathcal{G}, \mathbf{P}^{\prime}\right)$ )

$$
x_{t}=x+\int_{0}^{t} \sigma\left(y_{r}, z_{r}, x_{r}\right) d \beta_{r}+\int_{0}^{t} b\left(y_{r}, z_{r}, x_{r}\right) d r .
$$

We further recall that if $(\Omega, \mathcal{F}, \mathbf{P})$ is a probablity space on which $\left(w_{t}, \mathcal{F}_{t}\right)$ is a $d$ dimensional Brownian motion, then for any $s \geq 0, \mathcal{F}_{s}$ and $\sigma\left(w_{s+h}-w_{s}: h \geq 0\right)$ 
are $\mathbf{P}$-independent $\sigma$-algebras. In particular, for $0 \leq s \leq t, \mathcal{F}_{s}^{w}$ and $\mathcal{F}_{t-s}^{\theta_{s} w}$ are independent, where for $\omega \in \Omega$ and $h \in[0, t-s], \theta_{s} w(\omega)$ is the Brownian motion defined by $\theta_{s} w_{h}(\omega):=w_{s+h}(\omega)-w_{s}(\omega)$. Note that for any $\omega \in \Omega, w_{[0, s]}(\omega):=$ $\left.w \cdot\right|_{[0, s]}(\omega) \in C\left([0, s], E_{d}\right)$, while $\theta_{s} w_{[0, t-s]}(\omega) \in C\left([s, t], E_{d}\right)$.

Analogously, if $C:=C\left([0, \infty), E_{d}\right), \mathfrak{A}=\mathfrak{A}_{\infty}:=\sigma\left(x_{t}: t \geq 0\right)$, and $\mathcal{W}=$ $\mathcal{W}^{(d)}$ is the $d$-dimensional Wiener measure on $(C, \mathfrak{A})$, then $\left(x_{t}, \mathfrak{A}_{t}\right)$ is the canonical Brownian motion on $(C, \mathfrak{A}, \mathcal{W})$, where $\mathfrak{A}_{t}:=\sigma\left(x_{s}: 0 \leq s \leq t\right)$. (See $\S \S 2.2,2.3$ in [SV].) In particular, for $0 \leq s \leq t, \mathfrak{A}_{s}$ and $\mathfrak{A}_{t-s}^{\theta_{s} x}$ are $\mathcal{W}$-independent $\sigma$-algebras, where $\theta_{s} x_{h}:=x_{h+s}-x_{s}$ is also a Brownian motion under $\mathcal{W}$. We identify $C[0, t]$ with $C[0, s] \times C[s, t]$ under the map $\pi: C[0, t] \rightarrow C[0, s] \times C[s, t]$, defined by $\pi(x)=$. $\left(x .^{1}, x .^{2}\right):=\left(x_{[0, s]}, \theta_{s} x_{[0, t-s]}\right)$. Moreover, as a Brownian motion has independent increments, this map induces the identification $\mathcal{W}_{0, t}=\mathcal{W}_{0, s} \otimes \mathcal{W}_{s, t}$, where $\mathcal{W}_{0, s}$ and $\mathcal{W}_{s, t}$ denote the Wiener measures on $C[0, s], C[s, t]$, respectively.

The weak formulation of the differential game plays on the fact that admissible controls may be viewed as a.e. Borel functions of that space's Brownian motion (see p. 6 of [K1], and Lemma 1.5.6 in [N3]). Whether an expectation is taken with respect to $\mathbf{P}$ or $\mathcal{W}$ is therefore unimportant, as expectations with respect to $\mathbf{P}$ are expectations with respect to $\mathcal{W}$. For example, say that $y=\left\{y_{t}(\omega)\right\}$ is admissible and that $y_{t}(\omega)=\bar{y}_{t}\left(w_{[0, t]}(\omega)\right)$ for $\mathbf{P}$-a.e. $\omega \in \Omega$, for some Borel function $\bar{y}:[0, t] \times C[0, t] \rightarrow Y$. By the property of Wiener measure, for our fixed Brownian motion $w \cdot(\omega)$ on $\Omega$, we have $\mathcal{W}(B)=\mathbf{P}\left(w^{-1}(B)\right) \forall B \in \mathfrak{A}$, and hence $\mathcal{W}_{t}(B)=$ $\mathbf{P}\left(w_{[0, t]}^{-1}(B)\right) \forall B \in \mathfrak{A}_{t}$. Thus, for any function $f:(Y, \mathfrak{B}(Y)) \rightarrow\left(E_{1}, \mathfrak{B}\left(E_{1}\right)\right)$, we have

$$
\begin{aligned}
\mathbf{E}^{\mathbf{P}} f\left(y_{t}\right) & :=\int_{\Omega} f\left(y_{t}(\omega)\right) \mathbf{P}(d \omega) \\
& =\int_{\Omega} f\left(\bar{y}_{t}\left(w_{[0, t]}(\omega)\right)\right) \mathbf{P}(d \omega)=\int_{C[0, t]} f\left(\bar{y}_{t}(x .)\right) \mathbf{P} w_{[0, t]}^{-1}(d x .) \\
& =\int_{C[0, t]} f\left(\bar{y}_{t}(x .)\right) \mathcal{W}_{t}(d x .):=\mathbf{E}^{\mathcal{W}} f\left(\bar{y}_{t}\right) .
\end{aligned}
$$

Following the convention in [FN] and [N1, we use E to denote expectation, either with respect to $\mathbf{P}$ or $\mathcal{W}$.

In $\mathrm{FS}$, the authors prove the dynamic programming principle in the timeinhomogeneous setting, using a strong formulation of the differential game in the canonical space. In the time-homogeneous setting of [FN], and using the same definitions of control and strategy as in [FS], the authors prove a corresponding dynamic programming principle using a weak formulation of the game. It is interesting to note that the " $\epsilon$-optimal" strategies chosen in the "strong" proof of the dynamic programming principle in [FS] (see $(2.10),(2.11)$ p. 307) are identical to those chosen in the corresponding "weak" proof given in [FN] (see (5.14) p. 90, (5.24) p. 91 , with, of course, $\alpha, \beta$ and $\mathcal{M}, \mathcal{N}$ switched). The weak formulation of the game is also taken in the time-homogeneous setting in [N1]. Our formulation of the game is consistent with that of both [N1] and [FN].

Definition 3.1. An admissible control for player I (respectively II) is an $\mathcal{F}_{t}^{w}$ progressively measurable process $y_{t}(\omega)$ (respectively $z_{t}(\omega)$ ), having values in $Y$ (respectively $Z$ ). The set of all admissible controls for player I (respectively II) is denoted by $\mathcal{M}$ (respectively $\mathcal{N}$ ). We say that admissible controls $y^{1}, y^{2}$ in $\mathcal{M}$ are 
equal on $[s, t]$ if $\mathbf{P}\left\{\left|y_{r}^{1}-y_{r}^{2}\right|=0\right.$, a.e. $\left.r \in[s, t]\right\}=1$, with the analogous statement holding for controls in $\mathcal{N}$.

Definition 3.2. An admissible strategy for player I (respectively II) is a mapping $\alpha: \mathcal{N} \rightarrow \mathcal{M}$ (respectively $\beta: \mathcal{M} \rightarrow \mathcal{N}$ ) which preserves equality of controls. That is, if $z^{1}, z^{2} \in \mathcal{N}$ and $z^{1}=z^{2}$ on $[s, t]$, then $\alpha\left(z^{1}\right)=\alpha\left(z^{2}\right)$ on $[s, t]$. The set of all admissible strategies for player I (respectively II) is denoted by $\Gamma$ (respectively $\Delta$ ).

For simplicity, we suppose $t \in[0, T]$. Let $\pi=\left\{0=t_{0}<t_{1}<\cdots<t_{n}=T\right\}$ be a partition of $[0, T]$ and let $\|\pi\|=\max _{1 \leq i \leq n}\left(t_{i}-t_{i-1}\right)$ denote its mesh.

Definition 3.3. $y \in \mathcal{M}$ (respectively $z \in \mathcal{N}$ ) is a $\pi$-admissible control for player I (respectively II) if $y_{t}=y_{t_{j}}$ (respectively $\left.z_{t}=z_{t_{j}}\right) \forall t \in\left[t_{j}, t_{j+1}\right)$. The set of all $\pi$-admissible controls for player I (respectively II) is denoted by $\mathcal{M}_{\pi}$ (respectively $\left.\mathcal{N}_{\pi}\right)$.

Definition 3.4. $\alpha \in \Gamma$ is a $\pi$-admissible strategy for player I if $\alpha: \mathcal{N} \rightarrow \mathcal{M}_{\pi}$ has the further properties that (i) $\alpha(z)_{r}$ is $z$-independent for $r \in\left[0, t_{1}\right.$ ), and (ii) $z=\bar{z}$ on $\left[0, t_{j}\right)$ implies $\alpha(z)_{t_{j}}=\alpha(\bar{z})_{t_{j}}$. Corresponding defintions hold for $\pi$-admissible strategies $\beta: \mathcal{M} \rightarrow \mathcal{N}_{\pi}$ for player II. The set of all $\pi$-admissible strategies for player I (respectively II) is denoted by $\Gamma_{\pi}$ (respectively $\Delta_{\pi}$ ).

As in [FN], we define the $\pi$-upper value function $V_{\pi}^{+}$and $\pi$-lower value function $V_{\pi}^{-}$by

$$
\begin{gathered}
V_{\pi}^{+}(t) g(x)=\inf _{\beta \in \Delta_{\pi}} \sup _{y \in \mathcal{M}} J(t, x, y, \beta(y), g), \\
V_{\pi}^{-}(t) g(x)=\sup _{\alpha \in \Gamma_{\pi}} \inf _{z \in \mathcal{N}} J(t, x, \alpha(z), z, g),
\end{gathered}
$$

where $J(t, x, y, z, g)$ is as in (1.1). Taking $\alpha(\mathcal{N})=\hat{\mathcal{M}}$ and $\beta(\mathcal{M})=\hat{\mathcal{N}}$ in Corollary 1.3, along with the remark after Theorem 2.1, yields that $V_{\pi}^{+}(t) g, V_{\pi}^{-}(t) g \in C(\bar{D})$ and are continuous in $t$, for $g \in C(\bar{D})$. Moreover, $V_{\pi}^{+} g, V_{\pi}^{-} g$ are continuous in $t, x$ independent of the partition $\pi$ of $[0, T]$. We define the upper value function $V^{+}$ and lower value function $V^{-}$by

$$
\begin{aligned}
& V^{+}(t) g(x)=\inf _{\beta \in \cup_{n} \Delta_{\pi_{n}}} \sup _{y \in \mathcal{M}} J(t, x, y, \beta(y), g), \\
& V^{-}(t) g(x)=\sup _{\alpha \in \cup_{n} \Gamma_{\pi_{n}}} \inf _{z \in \mathcal{N}} J(t, x, \alpha(z), z, g) .
\end{aligned}
$$

Say that $\pi_{n} \leq \pi_{n+1}$; i.e., the set of partition points for $\pi_{n}$ is contained in the set of partition points for $\pi_{n+1}$. Then $\mathcal{N}_{\pi_{n}} \subset \mathcal{N}_{\pi_{n+1}}$, and hence $\Delta_{\pi_{n}} \subset \Delta_{\pi_{n+1}}$. The same holds for $\mathcal{M}_{\pi_{n}}$ and $\Gamma_{\pi_{n}}$. From this it follows that

$$
V^{+} g \leq V_{\pi_{n+1}}^{+} g \leq V_{\pi_{n}}^{+} g, \quad V_{\pi_{n}}^{-} g \leq V_{\pi_{n+1}}^{-} g \leq V^{-} g
$$

and hence

$$
V^{+}(t) g(x)=\lim _{n \rightarrow \infty} V_{\pi_{n}}^{+}(t) g(x), \quad V^{-}(t) g(x)=\lim _{n \rightarrow \infty} V_{\pi_{n}}^{-}(t) g(x) .
$$

It follows from our previous arguments that if $\pi_{n}<\pi_{n+1}$ with $\lim _{n \rightarrow \infty}\left\|\pi_{n}\right\|=0$, the value functions $V^{+}, V^{-}$do not depend on a sequence $\left\{\pi_{n}\right\}$.

Remark. As in [N1, we can describe $V^{+}$and $V^{-}$without reference to strategies. For example, for any fixed partition $\pi$, we can write

$$
V_{\pi}^{+}(t) g(x)=\inf _{\beta \in \Delta_{\pi}} \sup _{y \in \mathcal{M}} J(t, x, y, \beta(y), g)=\inf _{z \in \mathcal{N}_{\pi}} \sup _{y \in \mathcal{M}} J(t, x, y, z, g) .
$$


Indeed, $\forall \varepsilon>0, \exists \beta^{\varepsilon} \in \Delta_{\pi}$ with $\sup _{y \in \mathcal{M}} J\left(y, \beta^{\varepsilon}(y)\right)<\inf f_{\beta \in \Delta_{\pi}} \sup _{y \in \mathcal{M}} J(y, \beta(y))$ $+\varepsilon$. But $\forall y \in \mathcal{M}, \beta^{\varepsilon}(y) \in \mathcal{N}_{\pi}$; hence

$$
\inf _{z \in \mathcal{N}_{\pi}} \sup _{y \in \mathcal{M}} J(y, z)<\inf _{\beta \in \Delta_{\pi}} \sup _{y \in \mathcal{M}} J(y, \beta(y))+\varepsilon
$$

giving us one inequality. On the other hand, fix any $z \in \mathcal{N}_{\pi}$ and consider the constant strategy $\hat{\beta}: \mathcal{M} \rightarrow \mathcal{N}_{\pi}$ defined by $\hat{\beta}(y) \equiv z$. It is trivial to verify that $\hat{\beta} \in$ $\Delta_{\pi}$ and hence $\sup _{y \in \mathcal{M}} J(y, z)=\sup _{y \in \mathcal{M}} J(y, \hat{\beta}(y)) \geq \inf _{\beta \in \Delta_{\pi}} \sup _{y \in \mathcal{M}} J(y, \beta(y))$. Since $z \in \mathcal{N}_{\pi}$ was arbitrary, we have the other inequality. A similar argument yields

$$
V_{\pi}^{-}(t) g(x)=\sup _{\alpha \in \Gamma_{\pi}} \inf _{z \in \mathcal{N}} J(t, x, \alpha(z), z, g)=\sup _{y \in \mathcal{M}_{\pi}} \inf _{z \in \mathcal{N}} J(t, x, y, z, g) .
$$

Hence from (3.2) and (3.5), we can write, as in [N1,

$$
\begin{aligned}
V^{+}(t) g(x) & =\lim _{n \rightarrow \infty} \inf _{z \in \mathcal{N}_{\pi_{n}}} \sup _{y \in \mathcal{M}} J(t, x, y, z, g), \\
V^{-}(t) g(x) & =\lim _{n \rightarrow \infty} \sup _{y \in \mathcal{M}_{\pi_{n}}} \inf _{z \in \mathcal{N}} J(t, x, y, z, g) .
\end{aligned}
$$

We will use formulation (3.6) of the value functions in proving the semigroup property. We establish the semigroup property for $V^{+}(t)$ (the proof for $V^{-}(t)$ is similar), generalizing Theorem 2 in [N1], which corresponds to the case $D=E_{d}$, $\tau^{y, z, x}=+\infty$. Since value functions (as given by (3.5)) are independent of the sequence of partitions whose mesh tends to zero, we take for our $\pi_{n}$, the "approximate" partition of $[0, T]$, where $t_{j}=j 2^{-n}, 0 \leq j \leq\left[2^{n} T\right]+1$. We define $\mathcal{M}(n, j)$ to be the set of all $Y$-valued, $\mathcal{F}_{t}^{w}$-adapted processes defined on the interval $I(n, j):=\left[\frac{j}{2^{n}}, \frac{j+1}{2^{n}}\right] . \mathcal{N}(n, j)$ is defined analogously. We identify, for example, $\mathcal{N}$ with $\mathcal{N}(n, 0) \times \mathcal{N}(n, 1) \times \cdots$. We define the set of all constant strategies for player II on the interval $I(n, j)$ as

$$
\mathcal{N}_{c}(n, j)=\left\{z \in \mathcal{N}(n, j): z_{t}=z_{\frac{j}{2^{n}}}, \quad \forall t \in I(n, j)\right\} .
$$

Setting $y=\left(y^{0}, y^{1}, \ldots, y^{l}\right), z=\left(z^{0}, z^{1}, \ldots, z^{l}\right)$, where $y^{k}, z^{k} \in \mathcal{M}(n, k), \mathcal{N}_{c}(n, k)$ respectively, we define the lower value function $V^{+}$, for $l=\left[2^{n} T\right]$ by

$$
\begin{aligned}
V^{+}(t) g(x)= & \lim _{n \rightarrow \infty} \inf _{\mathcal{N}_{c}(n, 0)} \sup _{\mathcal{M}(n, 0)} \inf _{\mathcal{N}_{c}(n, 1)} \sup _{\mathcal{M}(n, 1)} \\
& \cdots \inf _{\mathcal{N}_{c}(n, l)} \sup _{\mathcal{M}(n, l)} J\left(t, x, y^{0} y^{1} \cdots y^{l}, z^{0} z^{1} \cdots z^{l}, g\right) .
\end{aligned}
$$

Under the identification $\mathcal{N}_{\pi_{n}}=\mathcal{N}_{c}(n, 0) \times \mathcal{N}_{c}(n, 1) \times \cdots \times \mathcal{N}_{c}(n, l)$, the above expression is exactly the expression for $V^{+}(t) g(x)$ given in (3.6) (see Theorem 1.4.1 in $[\mathrm{Fr}]$. As in [N1, to show the semigroup property for $V^{+}(t)$, set $\Delta=2^{-n}$ and define

$$
V_{n} g(x)=\inf _{z \in Z} \sup _{y \in \mathcal{M}} J(\Delta, x, y, z, g):=\inf _{z \in Z} S(\Delta, z) g(x) .
$$

By Bellman's principle, for any $z \in Z, S(t, z) g(x)$ is a semigroup on $C_{b}(D)$, for $0 \leq t \leq T$. Since $V_{n} g \in C_{b}(D)$ for $g \in C_{b}(D)$, we consider the set

$$
N(x)=\left\{z \in Z: V_{n} g(x)=S(\Delta, z) g(x)\right\} .
$$

By the continuity of $V_{n}$ and $S, N(x)$ is easily seen to be nonempty and compact. Hence, by Theorem 12.1.10 in [SV], a Borel selector $\bar{z}(\cdot)=\bar{z}(\cdot, \Delta, g)$ of $N(x)$ exists; i.e., $\bar{z}$ is a Borel function on $E_{d}$ and $\bar{z}(x) \in N(x)$. The following is the "stopped process" analogue of lemma 3.1 in [N1] (see $\S 10.2$ in [D]). 
Lemma 3.5. For $z^{j} \in \mathcal{N}_{c}(n, j)$ and $y^{j} \in \mathcal{M}(n, j), j=0, \ldots, k-1$, we have

$$
\begin{aligned}
& \inf _{z \in \mathcal{N}_{c}(n, k)} \sup _{y \in \mathcal{M}(n, k)} J\left((k+1) \Delta, x, y^{0} \cdots y^{k-1} y, z^{0} \cdots z^{k-1} z, g\right) \\
& \quad=J\left(k \Delta, x, y^{0} \cdots y^{k-1}, z^{0} \cdots z^{k-1}, V_{n} g\right) .
\end{aligned}
$$

Proof. Set $\hat{y}=y^{0} \cdots y^{k-1}, \hat{z}=z^{0} \cdots z^{k-1}$. Observe that for $\tilde{\mathcal{F}}_{k \Delta}:=\left\{A \in \mathcal{F}_{0}:\right.$ $\left.\left\{A, \tau^{\hat{y}, \hat{z}}>k \Delta\right\} \in \mathcal{F}_{k \Delta}\right\}:$

$$
\begin{aligned}
& \mathbf{E}_{x}^{\hat{y} y, \hat{z} z}\left\{\int_{k \Delta \wedge \tau}^{(k+1) \Delta \wedge \tau} f\left((\hat{y} y)_{r},(\hat{z} z)_{r}, x_{r}\right) e^{-\varphi_{r}} d r+g\left(x_{(k+1) \Delta \wedge \tau}\right) e^{-\varphi_{(k+1) \Delta \wedge \tau}} \mid \tilde{\mathcal{F}}_{k \Delta}\right\} \\
& =e^{-\varphi_{k \Delta \wedge \tau, \hat{y}, \hat{z}, x}} \mathbf{E}_{x_{k \Delta \wedge \tau, z}^{y, z}, \hat{z}, x}\left[\int_{0}^{\Delta \wedge \tau} f\left(y_{r}, z_{r}, x_{r}\right) e^{-\varphi_{r}} d r+g\left(x_{\Delta \wedge \tau}\right) e^{-\varphi_{\Delta \wedge \tau}}\right] \\
& =e^{-\varphi_{k \Delta \wedge \tau \hat{y}, \hat{z}, x}} J\left(\Delta, x_{k \Delta \wedge \tau, \hat{y}, \hat{z}, x}^{\hat{y}, y, z, g)}\right. \\
& \leq e^{-\varphi_{k \Delta \wedge \tau \hat{y}, \hat{z}, x}} S(\Delta, z) g\left(x_{k \Delta \wedge \tau, \hat{y}, \hat{y}, x}^{\hat{y}, x}\right),
\end{aligned}
$$

from which it follows that

$$
J((k+1) \Delta, x, \hat{y} y, \hat{z} z, g) \leq J(k \Delta, x, \hat{y}, \hat{z}, S(\Delta, z) g) .
$$

Since $y \in \mathcal{M}(n, k)$ was arbitrary,

$$
\sup _{y \in \mathcal{M}(n, k)} J((k+1) \Delta, x, \hat{y} y, \hat{z} z, g) \leq J(k \Delta, x, \hat{y}, \hat{z}, S(\Delta, z) g)
$$

hence

$$
\begin{aligned}
\inf _{z \in \mathcal{N}_{c}(n, k)} \sup _{y \in \mathcal{M}(n, k)} J((k+1) \Delta, x, \hat{y} y, \hat{z} z, g) & \leq \inf _{z \in \mathcal{N}_{c}(n, k)} J(k \Delta, x, \hat{y}, \hat{z}, S(\Delta, z) g) \\
& \leq J\left(k \Delta, x, \hat{y}, \hat{z}, V_{n} g\right),
\end{aligned}
$$

since $S(\Delta, z) g\left(x_{k \Delta \wedge \tau \hat{y}, \hat{z}, x}^{\hat{y}, \hat{z}, x}\right)=V_{n} g\left(x_{k \Delta \wedge \tau \hat{y}, \hat{z}, x}^{\hat{y}, x}\right)$ at $z=\bar{z}\left(x_{k \Delta \wedge \tau \hat{y}, \hat{z}, \hat{z}, x}^{\hat{y}, x}\right) \in \mathcal{N}_{c}(n, k)$.

We now show the other inequality. By the uniform continuity of $J(\Delta, x, y, z, g)$ in $x \in D, z \in Z$ (uniformly w.r.t. $y \in \mathcal{M}$ ), $\forall \varepsilon>0, \exists \delta(\varepsilon, g)>0$ s.t. $\left|x-x^{\prime}\right|<$ $\delta$ and $\left|z-z^{\prime}\right|<\delta$ imply

$$
\sup _{y \in \mathcal{M}}\left|J(\Delta, x, y, z, g)-J\left(\Delta, x^{\prime}, y, z^{\prime}, g\right)\right|<\frac{\varepsilon}{3} .
$$

Let $\left\{D_{n}\right\}_{n \geq 1}$ be a partition of $E_{d} \times Z$, with $\operatorname{diam}\left(D_{n}\right)<\delta$. Fix $\left(x_{n}, z_{n}\right) \in D_{n}$. By definition of $S\left(\Delta, z_{n}\right) g\left(x_{n}\right), \exists y_{n}^{*} \in \mathcal{M}(n, 0)$ s.t.

$$
S\left(\Delta, z_{n}\right) g\left(x_{n}\right)<J\left(\Delta, x_{n}, y_{n}^{*}, z_{n}, g\right)+\frac{\varepsilon}{3} .
$$

Since $y_{n}^{*} \in \mathcal{M}(n, 0), y_{n}^{*}$ is $w$-adapted. Moreover, since controls may be considered as a.e. Borel functions of the Brownian motion, there exists a Borel function $y_{n}:[0, \Delta] \times C\left([0, \Delta], E_{d}\right) \rightarrow Y$, which is progressively measurable and $y_{n}^{*}(t, \omega)=y_{n}(t, w \cdot(\omega))$ for P-a.e. $\omega \in \Omega$. Now define $\bar{y}=\bar{y}(\cdot, \Delta, g)$ by

$$
\bar{y}(t, w ., x, z)=\sum_{n} y_{n}(t, w .) I_{D_{n}}(x, z) .
$$

Then $\bar{y} \in \mathcal{M}(n, 0)$, and by (3.7), (3.8), for $(x, z) \in D \times Z$ (hence in $D_{n}$, for some $n$ ) and by the continuity of $S(\Delta, z) g(x)$ in $x \in D, z \in Z$,

$$
S(\Delta, z) g(x)<J(\Delta, x, \bar{y}, z, g)+\varepsilon .
$$


For $z \in \mathcal{N}_{c}(n, k)$, and $t \in I(n, k)$, define

$$
\bar{y}_{2}(t)=\bar{y}\left(t-k \Delta, \theta_{k \Delta} w ., x_{k \Delta}^{\hat{y}, \hat{z}, x}, z\right),
$$

where $\theta_{t} w_{s}=w_{s+t}-w_{t}$. Then $\bar{y}_{2} \in \mathcal{M}(n, k)$ and so

$$
\sup _{y \in \mathcal{M}(n, k)} J((k+1) \Delta, x, \hat{y} y, \hat{z} z, g) \geq J\left((k+1) \Delta, x, \hat{y} \bar{y}_{2}, \hat{z} z, g\right) \text {. }
$$

As before, and by (3.9),

$$
\begin{aligned}
& J\left((k+1) \Delta, x, \hat{y} y, \hat{z} \bar{z}_{2}, g\right) \\
& =\mathbf{E}\left[\int_{0}^{k \Delta \wedge \tau^{\hat{y}, \hat{z}, x}} f\left(\hat{y}_{r}, \hat{z}_{r}, x_{r}\right) e^{-\varphi_{r}} d r+J\left(\Delta, x_{k \Delta \wedge \tau \hat{y}, \hat{z}, x}^{\hat{y}, \hat{y}, x}, \bar{y}_{2}, z, g\right) e^{-\varphi_{k \Delta \wedge \tau \hat{y}, \hat{z}, x}}\right] \\
& \geq \mathbf{E}\left[\int_{0}^{k \Delta \wedge \tau^{\hat{y}, \hat{z}, x}} f\left(\hat{y}_{r}, \hat{z}_{r}, x_{r}\right) e^{-\varphi_{r}} d r+S(\Delta, z) g\left(x_{k \Delta \wedge \tau^{\hat{y}, \hat{z}, x}}^{\hat{y}, \hat{z}, x}\right) e^{-\varphi_{k \Delta \wedge \tau \hat{y}, \hat{z}, x}}\right]-\varepsilon \\
& \geq \mathbf{E}\left[\int_{0}^{k \Delta \wedge \tau^{\hat{y}, \hat{z}, x}} f\left(\hat{y}_{r}, \hat{z}_{r}, x_{r}\right) e^{-\varphi_{r}} d r+V_{n} g\left(x_{k \Delta \wedge \tau^{\hat{y}, \hat{z}, x}}^{\hat{y}, \hat{z}, x}\right) e^{-\varphi_{k \Delta \wedge \tau \hat{y}, \hat{z}, x}}\right]-\varepsilon \\
& =J\left(k \Delta, x, \hat{y}, \hat{z}, V_{n} g\right)-\varepsilon .
\end{aligned}
$$

Hence by (3.9), (3.10),

$$
\sup _{y \in \mathcal{M}(n, k)} J((k+1) \Delta, x, \hat{y} y, \hat{z} z, g) \geq J\left(k \Delta, x, \hat{y}, \hat{z}, V_{n} g\right)-\varepsilon .
$$

Since $z \in \mathcal{N}_{c}(n, k)$ was arbitrary, $\inf _{z \in \mathcal{N}_{c}(n, k)} \sup _{y \in \mathcal{M}(n, k)} J((k+1) \Delta, x, \hat{y} y, \hat{z} z, g) \geq$ $J\left(k \Delta, x, \hat{y}, \hat{z}, V_{n} g\right)-\varepsilon$. Letting $\varepsilon \rightarrow 0$, the lemma is proved.

Remark. Starting at the innermost $\inf _{z^{k} \in \mathcal{N}_{c}(n, k)} \sup _{y^{k} \in \mathcal{M}(n, k)}$ and working backwards, using Lemma 3.5 at each step gives

$$
\begin{gathered}
\inf _{z^{0} \in \mathcal{N}_{c}(n, 0)} \sup _{y^{0} \in \mathcal{M}(n, 0)} \cdots \inf _{z^{k} \in \mathcal{N}_{c}(n, k)} \sup _{y^{k} \in \mathcal{M}(n, k)} J\left((k+1) \Delta, x, y^{0} \cdots y^{k}, z^{0} \cdots z^{k}, g\right) \\
=J\left(0, x, y^{0}, z^{0}, V_{n}^{k+1} g\right)=V_{n}^{k+1} g(x) .
\end{gathered}
$$

Theorem 3.6. $V^{+}(t)$ is a semigroup on $C(\bar{D})$.

Proof. From the definition $V_{n} g(x)=\inf _{z \in Z} S\left(\frac{1}{2^{n}}, z\right) g(x), V_{n}$ is a monotone operator, i.e. $g \leq h \Rightarrow V_{n} g(x) \leq V_{n} h(x)$. From the semigroup properties of $S(t, z)$, $S\left(\frac{1}{2^{n-1}}, z\right) g(x)=S\left(\frac{1}{2^{n}}+\frac{1}{2^{n}}, z\right) g(x)=S\left(\frac{1}{2^{n}}, z\right)\left(S\left(\frac{1}{2^{n}}, z\right) g(x)\right) \geq V_{n}\left(S\left(\frac{1}{2^{n}}, z\right) g(x)\right)$ $\geq V_{n}\left(V_{n} g(x)\right)$, and hence $V_{n-1} g(x) \geq V_{n}^{2} g(x)$. We now define, for $t=\frac{k}{2^{n}}$,

$$
V_{n}(t) g(x):=V_{n}^{k} g(x) .
$$

For any binary $t, V_{n}(t) g$ is decreasing in $n$. This follows by induction on $k$. Indeed, for $k=1$, i.e. $t=\frac{1}{2^{n}}$,

$$
V_{n}(t) g(x)=V_{n} g(x) \geq V_{n+1}^{2} g(x):=V_{n+1}(t) g(x), \quad \text { for } t=\frac{2}{2^{n+1}}=\frac{1}{2^{n}} .
$$

Assume the statement is true for $k=m$. For $t=\frac{m+1}{2^{n}}$,

$$
\begin{aligned}
V_{n}(t) g(x) & =V_{n}^{m+1} g(x)=V_{n}^{m}\left(V_{n} g(x)\right) \geq V_{n+1}^{2 m}\left(V_{n} g(x)\right) \\
& \geq V_{n+1}^{2 m}\left(V_{n+1}^{2} g(x)\right)=V_{n+1}^{2 m+2} g(x)=V_{n+1}(t) g(x) .
\end{aligned}
$$


For $t=\frac{k}{2^{j}}, j \leq n, V_{j}^{k} g(x)=: V_{j}(t) g(x) \geq V_{n}(t) g(x)=: V_{n}^{k} g(x)$. Since $J$ is uniformly bounded for $g \in C_{b}(D)$, by (3.11) we have that $\left\{V_{n}(t) g: n \geq j\right\}$ is a totally bounded subset of $C_{b}(D)$, for $t=\frac{k}{2^{j}}$. Hence for any binary $t, \lim _{n \rightarrow \infty} V_{n}(t) g(x)$ exists. Set

$$
p(t, x, g)=\lim _{n \rightarrow \infty} V_{n}(t) g(x) .
$$

By the uniform continuity of $V^{+}(t) g$ on $[0, T], p(t, x, g)$ is continuous in $t$. By (3.11), the fact that $V_{n}(t) g(x)=V_{n}^{k+1} g(x)$, for $t=\frac{k+1}{2^{n}}$, and the definition of $V^{+}$, we have $p(t, x, g)=V^{+}(t) g(x)$ for binary $t$. By the continuity of $V^{+}(t) g(x)$ in $t$, $V^{+}(t) g(x)$ exists for any $t$. Observe that for binary $t, V_{n}(t) g$ is a semigroup for fixed $n$, since for $t=\frac{k}{2^{n}}, s=\frac{l}{2^{n}}$,

$$
V_{n}(t+s) g(x)=V_{n}^{k+l} g(x)=V_{n}^{k}\left(V_{n}^{l} g(x)\right)=V_{n}(s)\left(V_{n}(t) g(x)\right) .
$$

Hence for binary $t, s$,

$$
V^{+}(t+s) g(x)=\lim _{n \rightarrow \infty} V_{n}(t+s) g(x)=\lim _{n \rightarrow \infty} V_{n}(t)\left(V_{n}(s) g(x)\right) .
$$

Finally, from

$$
\begin{aligned}
\mid V_{n}(s) & \left(V_{n}(t) g(x)\right)-V^{+}(s)\left(V^{+}(t) g(x)\right) \mid \\
& \leq\left|V_{n}(s)\left(V_{n}(t) g(x)\right)-V_{n}(s)\left(V^{+}(t) g(x)\right)\right|+\left|V_{n}(s)\left(V^{+}(t) g(x)\right)-V(s)(V(t) g(x))\right| \\
& \leq\left\|V_{n}(t) g-V^{+}(t) g\right\|+\left\|V_{n}(s)\left(V^{+}(t) g\right)-V(s)(V(t) g)\right\|,
\end{aligned}
$$

we see that $\lim _{n \rightarrow \infty} V_{n}(s)\left(V_{n}(t) g(x)\right)=V^{+}(s)\left(V^{+}(t) g(x)\right)$, and hence by the above, for binary $t, s$,

$$
V^{+}(t+s) g(x)=V^{+}(t)\left(V^{+}(s) g(x)\right) .
$$

For arbitrary $t \geq 0$, set $\kappa_{n}(t)=2^{-n}\left[2^{n} t\right]$. Then, by the continuity of $V^{+}(t) g(x)$ in $t$,

$$
V^{+}(t+s) g(x)=\lim _{n \rightarrow \infty} V^{+}\left(\kappa_{n}(t)+\kappa_{n}(s)\right) g(x)=\lim _{n \rightarrow \infty} V^{+}\left(\kappa_{n}(t)\right)\left(V^{+}\left(\kappa_{n}(s)\right) g(x)\right)
$$

and again by $(*)$, we see that, for arbitrary $t, s \in[0, T]$,

$$
V^{+}(t+s) g(x)=V^{+}(t)\left(V^{+}(s) g(x)\right) .
$$

Hence by (3.6) and the remark, the function

$$
V^{+}(t) g(x)=\lim _{n \rightarrow \infty} \inf _{\beta \in \Delta_{\pi_{n}}} \sup _{y \in \mathcal{M}} J(t, x, y, \beta(z), g)
$$

is a semigroup on $C(\bar{D})$. As in Proposition 5.4 in [FN], we have

$$
\inf _{\beta \in \Delta_{\pi}} \sup _{y \in \mathcal{M}} J(t, x, y, \beta(y), g)=\sup _{\alpha \in \Gamma} \inf _{z \in \mathcal{N}_{\pi}} J(t, x, \alpha(z), z, g)
$$

and hence we can rewrite (3.12) as

$$
V^{+}(t) g(x)=\lim _{n \rightarrow \infty} \sup _{\alpha \in \Gamma} \inf _{z \in \mathcal{N}_{\pi_{n}}} J(t, x, \alpha(z), z, g)=\sup _{\alpha \in \Gamma} \inf _{z \in \mathcal{N}} J(t, x, \alpha(z), z, g) .
$$

A similar argument shows that the semigroup property is also satisfied by the lower value function

$$
V^{-}(t) g(x)=\lim _{n \rightarrow \infty} \inf _{\beta \in \Delta} \sup _{y \in \mathcal{M}_{\pi_{n}}} J(t, x, y, \beta(y), g)=\inf _{\beta \in \Delta} \sup _{y \in \mathcal{M}} J(t, x, y, \beta(y), g) .
$$


Hence by (1.7) in the remark following Theorem 1.4, we see that the upper and lower value functions

$$
\begin{aligned}
& v^{+}(x):=\sup _{\alpha \in \Gamma} \inf _{z \in \mathcal{N}} \mathbf{E}_{x}^{\alpha(z), z}\left[\int_{0}^{\tau} f\left(\alpha(z)_{r}, z_{r}, x_{r}\right) e^{-\varphi_{r}} d r+g\left(x_{\tau}\right) e^{-\varphi_{\tau}}\right], \\
& v^{-}(x):=\inf _{\beta \in \Delta} \sup _{y \in \mathcal{M}} \mathbf{E}_{x}^{y, \beta(y)}\left[\int_{0}^{\tau} f\left(y_{r}, \beta(y)_{r}, x_{r}\right) e^{-\varphi_{r}} d r+g\left(x_{\tau}\right) e^{-\varphi_{\tau}}\right]
\end{aligned}
$$

satisfy the dynamic programming principle. Here, $x_{t}^{\alpha(z), z, x}$ denotes the solution of $x_{t}=x+\int_{0}^{t} \sigma\left(y_{r}, z_{r}, x_{r}\right) d w_{r}+\int_{0}^{t} b\left(y_{r}, z_{r}, x_{r}\right) d r$ with $y=\alpha(z)$, and $\tau^{\alpha(z), z, x}=$ $\inf \left\{t \geq 0: x_{t}^{\alpha(z), z, x} \notin D\right\}$. Since $z \in \mathcal{N}, z_{t}$ is $\mathcal{F}_{t}$-adapted. Since $\alpha \in \Gamma, \alpha(z) \in \mathcal{M}$; hence $\alpha(z)_{t}$ is $\mathcal{F}_{t}$-adapted. Thus, $x_{t}^{\alpha(z), z, x}$ is $\mathcal{F}_{t}$-adapted and continuous in $t$. Since $D$ is open, $\tau^{\alpha(z), z, x}$ is a Markov time with respect to $\mathcal{F}_{t}$.

\section{INFINITESIMAL GENERATOR OF THE NONLINEAR SEMIGROUP AND ISAACS EQUATION}

Theorem 4.1. For $g \in C_{b}^{2}(D)$ and $x \in D$, define

$$
J(t) g(x)=\sup _{\alpha \in \Gamma} \inf _{z \in \mathcal{N}} \mathbf{E}_{x}^{\alpha, z}\left[\int_{0}^{t \wedge \tau} f\left(\alpha(z)_{r}, z_{r}, x_{r}\right) e^{-\varphi_{r}} d r+g\left(x_{t \wedge \tau}\right) e^{-\varphi_{t \wedge \tau}}\right] .
$$

Let $L(y, z, x) u:=\operatorname{tr}\left[a(y, z, x) u_{x x}\right]+b(y, z, x) \cdot u_{x}-c(y, z, x) u$. Then

$$
\lim _{t \rightarrow 0+} \frac{J(t) g(x)-g(x)}{t}=\min _{z \in Z} \max _{y \in Y}\{L(y, z, x) g(x)+f(y, z, x)\} .
$$

For convenience, let us denote $p(y, z, x):=L(y, z, x) g(x)+f(y, z, x)$ and $p(x):=$ $\min _{z \in Z} \max _{y \in Y} p(y, z, x)$. First we need a lemma.

Lemma 4.2. Given $\varepsilon>0$, there exists $\delta=\delta(\varepsilon, C, g)$ such that $\forall t<\delta, \forall y \in \mathcal{M}$ and $z \in \mathcal{N}$,

$$
\mathbf{E}_{x}^{y, z}\left|\int_{0}^{t \wedge \tau} p\left(y_{r}, z_{r}, x_{r}\right) e^{-\varphi_{r}} d r-\int_{0}^{t \wedge \tau} p\left(y_{r}, z_{r}, x\right) d r\right| \leq \varepsilon t .
$$

Proof. Since $g \in C_{b}^{2}(D)$, the coefficients and $f$ are uniformly bounded and $1-$ $e^{-\varphi_{r}^{y, z, x}} \leq \varphi_{r}^{y, z, x} \leq C r$, the left-hand side of (2) is majorized by

$$
\begin{aligned}
& \mathbf{E}_{x}^{y, z} \int_{0}^{t \wedge \tau}\left|p\left(y_{r}, z_{r}, x_{r}\right) e^{-\varphi_{r}}-p\left(y_{r}, z_{r}, x\right)\right| d r \\
& \leq \mathbf{E}_{x}^{y, z} \int_{0}^{t \wedge \tau}\left|p\left(y_{r}, z_{r}, x_{r}\right)\right|\left(1-e^{-\varphi_{r}}\right)+\left|p\left(y_{r}, z_{r}, x_{r}\right)-p\left(y_{r}, z_{r}, x\right)\right| d r \\
& \leq \mathbf{E}_{x}^{y, z} \int_{0}^{t \wedge \tau} C^{2}\left(\|g\|_{2}+1\right) r+\left|p\left(y_{r}, z_{r}, x_{r}\right)-p\left(y_{r}, z_{r}, x\right)\right| d r .
\end{aligned}
$$

Obviously, $C^{2}\left(\|g\|_{2}+1\right) t^{2}<C t \varepsilon$, provided $t<\delta_{1}(\varepsilon, C, g)$. To estimate the second integrand, we use the uniform (in $y, z$ ) Lipschitz continuity of the coefficients and $f$, as well as the uniform boundedness of the coefficients:

$$
\begin{aligned}
\left|p\left(y_{r}, z_{r}, x_{r}\right)-p\left(y_{r}, z_{r}, x\right)\right| & \leq\left|\operatorname{tr}\left[a\left(y_{r}, z_{r}, x_{r}\right) g_{x x}\left(x_{r}\right)\right]-\operatorname{tr}\left[a\left(y_{r}, z_{r}, x\right) g_{x x}(x)\right]\right| \\
& +\left|b\left(y_{r}, z_{r}, x_{r}\right) g_{x}\left(x_{r}\right)-b\left(y_{r}, z_{r}, x\right) g_{x}(x)\right| \\
& +\left|c\left(y_{r}, z_{r}, x_{r}\right) g\left(x_{r}\right)-c\left(y_{r}, z_{r}, x\right) g(x)\right| \\
& +\left|f\left(y_{r}, z_{r}, x_{r}\right)-f\left(y_{r}, z_{r}, x\right)\right| .
\end{aligned}
$$


The first 3 summands on the right are estimated in exactly the same way. For example, denoting $\gamma=(y, z)$,

$$
\begin{aligned}
& \left|\operatorname{tr}\left[a\left(\gamma_{r}, x_{r}\right) g_{x x}\left(x_{r}\right)\right]-\operatorname{tr}\left[a\left(\gamma_{r}, x\right) g_{x x}(x)\right]\right| \\
& \quad \leq\left\|a\left(\gamma_{r}, x_{r}\right)\right\|\left\|g_{x x}\left(x_{r}\right)-g_{x x}(x)\right\|+\left\|g_{x x}(x)\right\|\left\|a\left(\gamma_{r}, x_{r}\right)-a\left(\gamma_{r}, x\right)\right\| .
\end{aligned}
$$

Since $g \in C_{b}^{2}(D), \exists \delta=\delta(\varepsilon, g)$ such that $\forall x, x^{\prime} \in D,\left|x-x^{\prime}\right|<\delta$ implies that $\left\|g_{x x}(x)-g_{x x}\left(x^{\prime}\right)\right\|<\varepsilon$. Thus

$$
\begin{aligned}
& \mathbf{E}_{x}^{\gamma} \int_{0}^{t \wedge \tau}\left|\operatorname{tr}\left[a\left(\gamma_{r}, x_{r}\right) g_{x x}\left(x_{r}\right)\right]-\operatorname{tr}\left[a\left(\gamma_{r}, x\right) g_{x x}(x)\right]\right| d r \\
& \quad \leq C\left\{\mathbf{E}_{x}^{\gamma} \int_{0}^{t \wedge \tau}\left\|g_{x x}\left(x_{r}\right)-g_{x x}(x)\right\| d r+\left\|g_{x x}\right\|_{\infty} \mathbf{E}_{x}^{\gamma} \int_{0}^{t}\left|x_{r}-x\right| d r .\right\},
\end{aligned}
$$

and hence

$$
\begin{aligned}
\mathbf{E}_{x}^{\gamma} \int_{0}^{t \wedge \tau}\left\|g_{x x}\left(x_{r}\right)-g_{x x}(x)\right\| d r & \leq 2\left\|g_{x x}\right\|_{\infty} \mathbf{E}_{x}^{\gamma} \int_{0}^{t \wedge \tau} I_{\left|x_{r}-x\right| \geq \delta} d r+\varepsilon t \\
& \leq 2\left\|g_{x x}\right\|_{\infty} t \sup _{0 \leq r \leq t} \mathbf{P}_{x}^{\gamma}\left\{\left|x_{r}-x\right| \geq \delta\right\}+\varepsilon t .
\end{aligned}
$$

But for $0 \leq r \leq t, \mathbf{P}_{x}^{\gamma}\left\{\left|x_{r}-x\right| \geq \delta\right\} \leq \frac{\mathbf{E}_{x}^{\gamma}\left|x_{r}-x\right|}{\delta} \leq \frac{\sqrt{2} C \sqrt{r} e^{r}}{\delta} \leq \frac{\sqrt{2} C \sqrt{t} e^{t}}{\delta}$. Thus

$$
\begin{array}{r}
\mathbf{E}_{x}^{\gamma} \int_{0}^{t \wedge \tau}\left|\operatorname{tr}\left[a\left(\gamma_{r}, x_{r}\right) g_{x x}\left(x_{r}\right)\right]-\operatorname{tr}\left[a\left(\gamma_{r}, x\right) g_{x x}(x)\right]\right| d r \\
\leq C\left\{\left\|g_{x x}\right\|_{\infty} t \sqrt{t} e^{t} 3 C\left(\frac{1}{\delta}+1\right)+\varepsilon t\right\}<2 C t \varepsilon,
\end{array}
$$

provided $t<\delta_{2}(\varepsilon, C, g)$. Moreover, this same $\delta_{2}$ works for the next 2 summands. Finally

$$
\begin{aligned}
\mathbf{E}_{x}^{\gamma} \int_{0}^{t \wedge \tau}\left|f\left(\gamma_{r}, x_{r}\right)-f\left(\gamma_{r}, x\right)\right| d r & \leq C \mathbf{E}_{x}^{\gamma} \int_{0}^{t \wedge \tau}\left|x_{r}-x\right| d r \\
& \leq C \int_{0}^{t} \mathbf{E}_{x}^{\gamma}\left|x_{r}-x\right| d r \leq C^{2} \sqrt{2} t \sqrt{t} e^{t}<C t \varepsilon
\end{aligned}
$$

provided $t<\delta_{3}(\varepsilon, C)$. Hence the left-hand side of (3) is $\leq 8 C \varepsilon t$, for all $t<$ $\delta(\varepsilon, C, g):=\delta_{1} \wedge \delta_{2} \wedge \delta_{3}$, which proves (2).

Proof of Theorem 4.1. Since $g \in C^{2}(D)$, Itô's formula yields

$$
\mathbf{E}_{x}^{\alpha, z} g\left(x_{t \wedge \tau}\right) e^{-\varphi_{t \wedge \tau}}=g(x)+\mathbf{E}_{x}^{\alpha, z} \int_{0}^{t \wedge \tau} L\left(\alpha(z)_{r}, z_{r}, x_{r}\right) g\left(x_{r}\right) e^{-\varphi_{r}} d r .
$$

Thus

(3)

$$
J(t) g(x)-g(x)=\sup _{\alpha \in \Gamma} \inf _{z \in \mathcal{N}} \mathbf{E}_{x}^{\alpha, z} \int_{0}^{t \wedge \tau}\left(L\left(\alpha(z)_{r}, z_{r}, x_{r}\right) g\left(x_{r}\right)+f\left(\alpha(z)_{r}, z_{r}, x_{r}\right)\right) e^{-\varphi_{r}} d r .
$$


Recalling that $p(y, z, x):=L(y, z, x) g(x)+f(y, z, x)$ and $p(x):=\min _{z \in Z} \max _{y \in Y} p(y, z, x)$, by (3), $\forall \alpha \in \Gamma$ and $z \in \mathcal{N}$,

$$
\begin{aligned}
& \left|\frac{J(t) g(x)-g(x)}{t}-\min _{z \in Z} \max _{y \in Y}\{L(y, z, x) g(x)+f(y, z, x)\}\right| \\
& =\left|\sup _{\alpha \in \Gamma} \inf _{z \in \mathcal{N}} \frac{1}{t} \mathbf{E}_{x}^{\alpha, z} \int_{0}^{t \wedge \tau} p\left(\alpha(z)_{r}, z_{r}, x_{r}\right) e^{-\varphi_{r}} d r-p(x)\right| \\
& \leq \mid \sup _{\alpha \in \Gamma} \inf _{z \in \mathcal{N}} \frac{1}{t} \mathbf{E}_{x}^{\alpha, z} \int_{0}^{t \wedge \tau} p\left(\alpha(z)_{r}, z_{r}, x_{r}\right) e^{-\varphi_{r}} d r \\
& -\sup _{\alpha \in \Gamma} \inf _{z \in \mathcal{N}} \frac{1}{t} \mathbf{E}_{x}^{\alpha, z} \int_{0}^{t \wedge \tau} p\left(\alpha(z)_{r}, z_{r}, x\right) d r \mid \\
& +\left|\sup _{\alpha \in \Gamma} \inf _{z \in \mathcal{N}} \frac{1}{t} \mathbf{E}_{x}^{\alpha, z} \int_{0}^{t \wedge \tau} p\left(\alpha(z)_{r}, z_{r}, x\right) d r-p(x)\right| \\
& \leq \frac{1}{t} \sup _{y \in \mathcal{M}} \sup _{z \in \mathcal{N}} \mathbf{E}_{x}^{y, z}\left|\int_{0}^{t \wedge \tau} p\left(y_{r}, z_{r}, x_{r}\right) e^{-\varphi_{r}} d r-\int_{0}^{t \wedge \tau} p\left(y_{r}, z_{r}, x\right) d r\right| \\
& +\left|\sup _{\alpha \in \Gamma} \inf _{z \in \mathcal{N}} \frac{1}{t} \mathbf{E}_{x}^{\alpha, z} \int_{0}^{t \wedge \tau} p\left(\alpha(z)_{r}, z_{r}, x\right) d r-p(x)\right| .
\end{aligned}
$$

By Lemma 4.1, we need only estimate the last summand in (4). Fix any constant $\bar{z} \in Z$ and consider the constant control $z_{t} \equiv \bar{z}$. For any $\alpha \in \Gamma$,

$$
\begin{aligned}
\inf _{z \in \mathcal{N}} & \frac{1}{t} \mathbf{E}_{x}^{\alpha, z} \int_{0}^{t \wedge \tau} p\left(\alpha(z)_{r}, z_{r}, x\right) d r \\
& \leq \frac{1}{t} \mathbf{E}_{x}^{\alpha, \bar{z}} \int_{0}^{t \wedge \tau} p\left(\alpha(\bar{z})_{r}, \bar{z}, x\right) d r \leq \max _{y \in Y} p(y, \bar{z}, x) \mathbf{E}\left(\frac{t \wedge \tau^{\alpha(\bar{z}), \bar{z}, x}}{t}\right) \\
& =-\max _{y \in Y} p(y, \bar{z}, x)\left[1-\mathbf{E}_{x}^{\alpha, \bar{z}}\left(\frac{t \wedge \tau}{t}\right)\right]+\max _{y \in Y} p(y, \bar{z}, x) \\
& \leq \tilde{C}\left[1-\mathbf{E}_{x}^{\alpha, \bar{z}}\left(\frac{t \wedge \tau}{t}\right)\right]+\max _{y \in Y} p(y, \bar{z}, x) .
\end{aligned}
$$

Since $\bar{z} \in Z$ was arbitrary, taking $\inf _{Z}$ and using the inequality $\inf \left(h^{1}+h^{2}\right) \leq$ $\sup h^{1}+\inf h^{2}$ yields

$$
\begin{aligned}
\inf _{z \in \mathcal{N}} \frac{1}{t} \mathbf{E}_{x}^{\alpha, z} \int_{0}^{t \wedge \tau} p\left(\alpha(z)_{r}, z_{r}, x\right) d r-p(x) & \leq \tilde{C} \sup _{\bar{z} \in Z}\left[1-\mathbf{E}_{x}^{\alpha, \bar{z}}\left(\frac{t \wedge \tau}{t}\right)\right] \\
& \leq \tilde{C}\left[1-\inf _{z \in \mathcal{N}} \mathbf{E}_{x}^{\alpha, z}\left(\frac{t \wedge \tau}{t}\right)\right],
\end{aligned}
$$

where $\tilde{C}=C\left(\|g\|_{2, D}+1\right)$. Recall that if $x \in D$ with $\operatorname{dist}(x, \partial D) \geq \kappa$ and for any $(y, z) \in \mathcal{M} \times \mathcal{N}$, for $t>0$ sufficiently small (depending only on $\kappa,|b|_{\infty}$ ), there exists a constant $N$, depending only on $\|\sigma\|_{\infty}$, such that

$$
\mathbf{P}\left\{\tau^{y, z, x} \leq t\right\} \leq \frac{N \cdot t^{2}}{\kappa^{4}} .
$$

Fix $x \in D, z \in \mathcal{N}, \alpha \in \Gamma$ and choose $t>0$ so small that (6) holds,

$$
\mathbf{E}_{x}^{\alpha, z}\left(\frac{t \wedge \tau}{t}\right) \geq \mathbf{P}_{x}^{\alpha, z}\{\tau>t\} \geq 1-\frac{N \cdot t^{2}}{\kappa^{4}}
$$


and hence

$$
1-\inf _{z \in \mathcal{N}} \mathbf{E}_{x}^{\alpha, z}\left(\frac{t \wedge \tau}{t}\right) \leq \frac{N \cdot t^{2}}{\kappa^{4}} .
$$

In particular, if $x \in K$, where $K \subset D$ is compact with $\operatorname{dist}(K, \partial D)>0$, we can take $\kappa=\operatorname{dist}(K, \partial D)$ in (7). Since $\alpha \in \Gamma$ was arbitrary, (5) and (7) yield

$$
\sup _{\alpha \in \Gamma} \inf _{z \in \mathcal{N}} \frac{1}{t} \mathbf{E}_{x}^{\alpha, z} \int_{0}^{t \wedge \tau} p\left(\alpha(z)_{r}, z_{r}, x\right) d r-p(x) \leq \frac{\tilde{C} N \cdot t^{2}}{\kappa^{4}}<\varepsilon .
$$

for $t<\delta(\varepsilon, \kappa, g, C)$. On the other hand, for any strategy $\alpha \in \Gamma$, we obviously have

$$
\sup _{\alpha \in \Gamma} \inf _{z \in \mathcal{N}} \frac{1}{t} \mathbf{E}_{x}^{\alpha, z} \int_{0}^{t \wedge \tau} p\left(\alpha(z)_{r}, z_{r}, x\right) d r \geq \inf _{z \in \mathcal{N}} \frac{1}{t} \mathbf{E}_{x}^{\alpha, z} \int_{0}^{t \wedge \tau} p\left(\alpha(z)_{r}, z_{r}, x\right) d r .
$$

Following [ES], [FS], fix $x \in D$ and set $p(x):=\min _{z \in Z} \max _{y \in Y} p(y, z, x)$. Then $\forall z \in Z, \exists y=y(z) \in Y$ for which $p(x) \leq p(y(z), z, x)$. Since $p(y, \cdot, x)$ is continuous and $Z$ is compact, $p(y, \cdot, x)$ is uniformly continuous in $Z$. Hence $\forall \varepsilon>0, \exists \delta(\epsilon)$ so that $\forall \zeta, z \in Z,|\zeta-z|<\delta$ implies $|p(y, \zeta, x)-p(y, z, x)|<\varepsilon$. In particular, for $z \in Z$ and the $y=y(z) \in Y$ described above, if $\zeta \in Z$ with $|\zeta-z|<\delta$, then $p(x)-\varepsilon<p(y(z), \zeta, x)$. But $Z$ is compact, so we can find $r_{1}, \ldots, r_{n}>$ $0, z_{1}, \ldots, z_{n} \in Z$ with $Z \subset \bigcup_{i=1}^{n} B_{r_{i}}\left(z_{i}\right), r_{i}<\delta$. Hence $\forall \zeta \in Z$, if $\left|\zeta-z_{i}\right|<r_{i}$, then $p(x)-\varepsilon<p\left(y_{i}, \zeta, x\right)$, where $y_{i}=y_{i}\left(z_{i}\right)$ is as above. Now define a map $\Phi: Z \rightarrow Y$ by $\Phi(z)=y_{k}$ if $z \in B_{r_{k}}\left(z_{k}\right) \backslash \bigcup_{i<k} B_{r_{i}}\left(z_{i}\right)$ for $k=1, \ldots, n$. Then for all $z \in Z, p(x)-\varepsilon<p(\Phi(z), z, x)$. Now define $\alpha^{\varepsilon}: \mathcal{N} \rightarrow \mathcal{M}$ by $\alpha^{\varepsilon}(z)_{r}=\Phi\left(z_{r}\right)$ for $z \in \mathcal{N}$. Then $\alpha^{\varepsilon} \in \Gamma$. That is, for $z \in \mathcal{N}, \alpha^{\varepsilon}(z) \in \mathcal{M}$, since $\forall B \in \mathfrak{B}(Y)$, $\left(\alpha^{\varepsilon}(z)_{r}\right)^{-1}(B)=z_{r}^{-1}\left(\Phi^{-1}(B)\right) \in \mathcal{F}_{r}$, since $\Phi$ is Borel measurable and $z_{t}$ is $\mathcal{F}_{t^{-}}$ adapted. Then for any $z \in \mathcal{N}$ and any $t>0$, for $0 \leq r \leq t$, we have

$$
p(x)-\varepsilon<p\left(\alpha^{\varepsilon}(z)_{r}, z_{r}, x\right),
$$

which immediately gives

$$
(p(x)-\varepsilon) \mathbf{E}_{x}^{\alpha^{\varepsilon}, z}\left(\frac{t \wedge \tau}{t}\right) \leq \frac{1}{t} \mathbf{E}_{x}^{\alpha^{\varepsilon}, z} \int_{0}^{t \wedge \tau} p\left(\alpha^{\varepsilon}(z)_{r}, z_{r}, x\right) d r .
$$

This yields

$$
\begin{aligned}
-\tilde{C}\left[1-\mathbf{E}_{x}^{\alpha^{\varepsilon}, z}\left(\frac{t \wedge \tau}{t}\right)\right] & \leq(\varepsilon-p(x))\left[1-\mathbf{E}_{x}^{\alpha^{\varepsilon}, z}\left(\frac{t \wedge \tau}{t}\right)\right] \\
& \leq \frac{1}{t} \mathbf{E}_{x}^{\alpha^{\varepsilon}, z} \int_{0}^{t \wedge \tau} p\left(\alpha^{\varepsilon}(z)_{r}, z_{r}, x\right) d r-p(x)+\varepsilon .
\end{aligned}
$$

Taking $\inf _{z \in \mathcal{N}}$ and then using (9) yields

$$
-\tilde{C}\left[1-\inf _{z \in \mathcal{N}} \mathbf{E}_{x}^{\alpha^{\varepsilon}, z}\left(\frac{t \wedge \tau}{t}\right)\right] \leq \sup _{\alpha \in \Gamma} \inf _{z \in \mathcal{N}} \frac{1}{t} \mathbf{E}_{x}^{\alpha, z} \int_{0}^{t \wedge \tau} p\left(\alpha(z)_{r}, z_{r}, x\right) d r-p(x)+\varepsilon .
$$

By (7), we get (again taking $\kappa=\operatorname{dist}(K, \partial D)$ ),

$$
\frac{-\tilde{C} 2 N \cdot t^{2}}{\kappa^{4}} \leq \sup _{\alpha \in \Gamma} \inf _{z \in \mathcal{N}} \frac{1}{t} \mathbf{E}_{x}^{\alpha, z} \int_{0}^{t \wedge \tau} p\left(\alpha(z)_{r}, z_{r}, x\right) d r-p(x)+\varepsilon .
$$

Along with (8), this immediately implies that for $t<\delta(\varepsilon, \kappa, g, C)$ (the same one as above),

$$
\left|\sup _{\alpha \in \Gamma} \inf _{z \in \mathcal{N}} \frac{1}{t} \mathbf{E}_{x}^{\alpha, z} \int_{0}^{t \wedge \tau} p\left(\alpha(z)_{r}, z_{r}, x\right) d r-p(x)\right|<2 \varepsilon .
$$


This, (2) and (4) imply that for any $x \in D$ with $\operatorname{dist}(x, \partial D)>0$ and any $\varepsilon>0$, $\exists \delta=\delta(\varepsilon, \kappa, g, C)$ such that for $t<\delta$,

$$
\left|\frac{J(t) g(x)-g(x)}{t}-\min _{z \in Z} \max _{y \in Y}\{L(y, z, x) g(x)+f(y, z, x)\}\right|<3 \varepsilon .
$$

Theorem 4.3. The upper value function $v^{+}$is a viscosity solution of the upper Isaacs equation

$$
F^{+}[u](x):=\min _{z \in Z} \max _{y \in Y}\{L(y, z, x) u(x)+f(y, z, x)\}=0 \quad \text { in } D,
$$

and the lower value function $v^{-}$is a viscosity solution of the lower Isaacs equation

$$
F^{-}[u](x):=\max _{y \in Y} \min _{z \in Z}\{L(y, z, x) u(x)+f(y, z, x)\}=0 \quad \text { in } D,
$$

where $L(y, z, x) u:=\operatorname{tr}\left[a(y, z, x) u_{x x}\right]+b(y, z, x) \cdot u_{x}-c(y, z, x) u$.

Proof. Since $g \in C(\bar{D})$, both $v^{+}, v^{-} \in C(\bar{D})$, by Theorem 2.1. We prove the theorem only for $v^{+}$, since the proof for $v^{-}$is similar. So set

$$
v(x)=v^{+}(x):=\sup _{\alpha \in \Gamma} \inf _{z \in \mathcal{N}} \mathbf{E}_{x}^{\alpha, z}\left[\int_{0}^{\tau} f\left(\alpha(z)_{r}, z_{r}, x_{r}\right) e^{-\varphi_{r}} d r+g\left(x_{\tau}\right) e^{-\varphi_{\tau}}\right] .
$$

First, we show that $v$ is a subsolution. Fix $x_{0} \in D$ with $\operatorname{dist}\left(x_{0}, \partial D\right) \geq \delta$. Since $v \in C(D)$, let $\psi \in C^{2}(D)$ such that $v-\psi$ has a local maximum at $x_{0}$. We want to show $F^{+}[\psi]\left(x_{0}\right) \geq 0$. Without loss of generality, we may assume this local maximum is a global maximum and $v\left(x_{0}\right)=\psi\left(x_{0}\right)$. That is, $v(x) \leq \psi(x)$ in $D$ and $v\left(x_{0}\right)=\psi\left(x_{0}\right)$. By the dynamic programming principle, for any $h>0$ we have

$$
\begin{aligned}
\psi\left(x_{0}\right)=v\left(x_{0}\right) & =\sup _{\alpha \in \Gamma} \inf _{z \in \mathcal{N}} \mathbf{E}_{x_{0}}^{\alpha, z}\left[\int_{0}^{\tau \wedge h} f\left(\alpha(z)_{r}, z_{r}, x_{r}\right) e^{-\varphi_{r}} d r+v\left(x_{\tau \wedge h}\right) e^{-\varphi_{\tau \wedge h}}\right] \\
& \leq \sup _{\alpha \in \Gamma} \inf _{z \in \mathcal{N}} \mathbf{E}_{x_{0}}^{\alpha, z}\left[\int_{0}^{\tau \wedge h} f\left(\alpha(z)_{r}, z_{r}, x_{r}\right) e^{-\varphi_{r}} d r+\psi\left(x_{\tau \wedge h}\right) e^{-\varphi_{\tau \wedge h}}\right],
\end{aligned}
$$

where for any $z \in \mathcal{N}$ and $\alpha \in \Gamma, x_{t}^{\alpha(z), z, x_{0}}$ is a solution of

$$
x_{t}=x_{0}+\int_{0}^{t} \sigma\left(y_{r}, z_{r}, x_{r}\right) d w_{r}+\int_{0}^{t} b\left(y_{r}, z_{r}, x_{r}\right) d r
$$

with $y=\alpha(z)$, and $\tau^{\alpha(z), z, x_{0}}=\inf \left\{t \geq 0: x_{t}^{\alpha(z), z, x_{0}} \notin D\right\}$. By Itô's formula,

$$
\mathbf{E}_{x_{0}}^{\alpha, z} \psi\left(x_{\tau \wedge h}\right) e^{-\varphi_{\tau \wedge h}}=\psi\left(x_{0}\right)+\mathbf{E}_{x_{0}}^{\alpha, z} \int_{0}^{\tau \wedge h} L\left(\alpha(z)_{r}, z_{r}, x_{r}\right) \psi\left(x_{r}\right) e^{-\varphi_{r}} d r,
$$

and hence by (1),

$$
0 \leq \sup _{\alpha \in \Gamma} \inf _{z \in \mathcal{N}} \mathbf{E}_{x_{0}}^{\alpha, z} \int_{0}^{\tau \wedge h}\left[f\left(\alpha(z)_{r}, z_{r}, x_{r}\right)+L\left(\alpha(z)_{r}, z_{r}, x_{r}\right) \psi\left(x_{r}\right)\right] e^{-\varphi_{r}} d r .
$$

Denoting the above expectation by $q\left(h, \alpha(z), z, x_{0}\right),(2)$ gives that

$$
0 \leq \sup _{\alpha \in \Gamma} \inf _{z \in \mathcal{N}} q\left(h, \alpha(z), z, x_{0}\right) .
$$

But for any $\alpha \in \Gamma$ and $z \in \mathcal{N}, \alpha(z) \in \mathcal{M}$. Fix any constant $\bar{z} \in Z$ and recall $Z \subset \mathcal{N}$. For any $\alpha \in \Gamma$, we have

$$
\inf _{z \in \mathcal{N}} q\left(h, \alpha(z), z, x_{0}\right) \leq q\left(h, \alpha(\bar{z}), \bar{z}, x_{0}\right) \leq \sup _{y \in \mathcal{M}} q\left(h, y, \bar{z}, x_{0}\right)
$$


and since $\alpha \in \Gamma$ was arbitrary, we have $0 \leq \sup _{\alpha \in \Gamma} \inf _{z \in \mathcal{N}} q\left(h, \alpha(z), z, x_{0}\right) \leq$ $\sup _{y \in \mathcal{M}} q\left(h, y, \bar{z}, x_{0}\right)$. For convenience, set $p(y, z, x):=L(y, z, x) \psi(x)+f(y, z, x)$. Then by (2), since $h>0$,

$0 \leq \sup _{y \in \mathcal{M}} \mathbf{E}_{x_{0}}^{y, \bar{z}} \frac{1}{h} \int_{0}^{\tau \wedge h} p\left(y_{r}, \bar{z}, x_{r}\right) e^{-\varphi_{r}} d r \leq \sup _{y \in \mathcal{M}} \mathbf{E}_{x_{0}}^{y, \bar{z}} \frac{1}{h} \int_{0}^{\tau \wedge h} \max _{y \in Y} p\left(y, \bar{z}, x_{r}\right) e^{-\varphi_{r}} d r$

where $x_{t}^{y, \bar{z}, x_{0}}$ is a solution of $x_{t}=x_{0}+\int_{0}^{t} \sigma\left(y_{r}, \bar{z}, x_{r}\right) d w_{r}+\int_{0}^{t} b\left(y_{r}, \bar{z}, x_{r}\right) d r$ and $\tau^{y, \bar{z}, x_{0}}=\inf \left\{t \geq 0: x_{t}^{y, \bar{z}, x_{0}} \notin D\right\}$. By the same techniques as in Theorem 4.1, letting $h \rightarrow 0+$ in (3) yields $0 \leq \max _{y \in Y} p\left(y, \bar{z}, x_{0}\right)$, and since $\bar{z} \in Z$ was arbitrary,

$$
0 \leq \min _{z \in Z} \max _{y \in Y} p\left(y, z, x_{0}\right)=F^{+}[\psi]\left(x_{0}\right)
$$

and so $v$ is a subsolution. The proof that $v$ is a supersolution of $F^{+}[v]=0$ proceeds the same way and uses an argument similar to the argument of Theorem 4.1 following (4.9).

In addition to the assumptions $(1.1)^{\prime}$, our results have been derived under the assumption of the existence of a global barrier. It is well known that if $\partial D$ satisfies a uniform exterior sphere condition and $a=\frac{1}{2} \sigma \sigma^{*}$ is nondegenerate, such a barrier exists. (See the discussion following Assumption 1.0.) For Isaacs equations with uniformly bounded and uniformly (in $y, z$ ) Lipschitz coefficients, continuous viscosity solutions of the Dirichlet problem with continuous boundary values are unique (see III.1, V.1 of [IL]). Existence and uniqueness of continuous viscosity solutions also holds (see Theorem II.2 in IL]) in the degenerate case when $\partial D \in C^{2}$, $\inf _{y, z, x} c(y, z, x)=c_{0}>0$, and $\inf _{y, z}\langle a(y, z, x) n(x), n(x)\rangle>0$ on $\partial D$, where $n(x)$ is the outward unit normal to $D$ at $x \in \partial D$. Since we've shown that $v^{+}, v^{-} \in C(\bar{D})$, for arbitrary $g \in C(\bar{D})$, the following result is an immediate consequence of Proposition II.1 in IL.

Theorem 4.4. In addition to $(1.1)^{\prime}$, suppose that either (i) $\partial D$ satisfies a uniform exterior sphere condition and $a=\frac{1}{2} \sigma \sigma^{*}$ is nondegenerate or (ii) $\partial D \in C^{2}$, the equation is degenerate elliptic, $\inf _{y, z, x} c(y, z, x) \geq c_{0}>0, \inf _{y, z}\langle a(y, z, x) n(x), n(x)\rangle$ $>0$ on $\partial D$, and a global barrier exists. Then the upper value function $v^{+}$is the unique viscosity solution of the Dirichlet problem for the upper Isaacs equation

$$
\left\{\begin{aligned}
F^{+}[v](x):=\min _{z \in Z} \max _{y \in Y}\{L(y, z, x) v(x)+f(y, z, x)\} & =0 & & \text { in } D, \\
& v=g & & \text { on } \partial D
\end{aligned}\right.
$$

and the lower value function $v^{-}$is the unique viscosity solution of the Dirichlet problem for the lower Isaacs equation

$$
\left\{\begin{aligned}
F^{-}[v](x):=\max _{y \in Y} \min _{z \in Z}\{L(y, z, x) v(x)+f(y, z, x)\} & =0 & & \text { in } D, \\
v & =g & & \text { on } \partial D
\end{aligned}\right.
$$


for arbitrary $g \in C(\bar{D})$, where $L(y, z, x) u:=\operatorname{tr}\left[a(y, z, x) u_{x x}\right]+b(y, z, x) \cdot u_{x}-$ $c(y, z, x) u$.

Corollary 4.5. Under either of the assumptions of Theorem 4.4, if the Isaacs min-max condition is satisfied, that is, if

$$
F^{+}(m, p, r, x)=F^{-}(m, p, r, x) \quad \forall(m, p, r, x) \in S_{d} \times \mathbb{R}^{d} \times \mathbb{R} \times D,
$$

then $v^{+}=v^{-}$in $D$ and the differential game has value.

\section{REFERENCES}

[BL] A. Bensoussan and J.L. Lions, Applications des Inéquations Variationnelles en Contrôle Stochastique, Dunod, Paris, 1978. MR 0513618 (58:23923)

[CC1] L. Caffarelli and X. Cabre, Fully Nonlinear Elliptic Equations, Amer. Math. Soc., Providence, R.I., 1995. MR1351007(96h:35046)

[CC2] _ Interior $C^{2, \alpha}$ Regularity Theory for a Class of Nonconvex Fully Nonlinear Elliptic Equations, J. Math. Pures Appl. 82 (2003), 573-612. MR1995493 (2004f:35049)

[D] E.B. Dynkin, Markov Processes, Vol. 1, Berlin, 1965, English transl. Grundl. Math. Wiss. Vol. 121, Springer-Verlag. MR0193671 (33:1887)

[ES] L.C. Evans and P.E. Souganidis, Differential Games and Representation Formulas for Solutions of Hamilton-Jacobi-Isaacs Equations, Indiana Univ. Math. J. 33 (1984), 773797. MR756158 (86d:90185)

[FN] W.H. Fleming and M. Nisio, Differential Games for Stochastic Partial Differential Equations, Nagoya Math. J. 131 (1993), 75-107. MR.1238634(94h:93074)

[FS] W.H. Fleming and P.E. Souganidis, On the Existence of Value Functions of Two-Player, Zero-Sum Stochastic Differential Games, Indiana Univ. Math. J. 38 (1989), 293-314. MR997385 (90e:93089)

[Fr] A. Friedman, Differential Games, Wiley, New York, 1971. MR0421700 (54:9696)

[GT] D. Gilbarg and N.S. Trudinger, Elliptic Partial Differential Equations of Second Order, 2nd ed., Springer-Verlag, New York, 1983. MR737190 (86c:35035)

[I] R. Isaacs, Differential Games, Wiley, New York, 1965. MR0210469 (35:1362)

[IL] H. Ishii and P.L. Lions, Viscosity Solutions of Fully Nonlinear Second-Order Elliptic Partial Differential Equations, J. Diff. Equat. 83 (1990), 26-78. MR1031377 (90m:35015)

[Ish] H. Ishii, On Uniqueness and Existence of Viscosity Solutions of Fully Nonlinear SecondOrder Elliptic PDEs, Comm. Pure Appl. Math. 42 (1989), 14-45. MR.973743 (89m:35070)

[Ka] M. A. Katsoulakis, A Representation Formula and Regularizing Properties for Viscosity Solutions of Second-Order Fully Nonlinear Degenerate Parabolic Equations, Nonlinear Analysis 24 (1995), 147-158. MR:1312585 (95m:35039)

[K1] N.V. Krylov, Controlled Diffusion Processes, Nauka, Moscow, 1977, English transl. Springer-Verlag, New York, 1980. MR601776 (82a:60062)

[K2] Smoothness of the Payoff Function for a Controlled Diffusion Process in a Domain, vol. 34, Izv. Acad. Nauk. SSSR Ser. Mat., 1990, pp. 65-95, English transl. in Math. USSR Izv. MR992979 (90f:93040)

[K3] , On Controlled Diffusion Processes with Unbounded Coefficients, vol. 19, Izv. Acad. Nauk. SSSR Ser. Mat., 1982, pp. 41-64, English transl. in Math. USSR Izv..

[L] P.L. Lions, Optimal Control of Diffusion Processes and Hamilton-Jacobi-Bellman Equations. Part 1: The Dynamic Programming Principle and Applications, Communicatons in PDE. 8 (1983), 1101-1174. MR709164 (85i:49043a)

[LM1] P.L. Lions and J.L. Menaldi, Optimal Control of Stochastic Integrals and Hamilton-JacobiBellman Equations I, II, Siam J. Control and Optimization, No. 1, 20, (1982), 58-81, 82-95. MR642179 (83c:49039)

[N1] M. Nisio, Stochastic Differential Games and Viscosity Solutions of Isaacs Equations, vol. 110, Nagoya Math. J., 1988, pp. 163-184. MR945913 (90b:93100)

[N2] - Some Remarks on Stochastic Optimal Control, Proceedings of 3rd USSR-Japan Symposium on Probability Theory, Springer-Verlag, 1976, pp. 446-460. MR0439373 $(55: 12266)$

[N3] , Stochastic Control Theory, ISI Lecture Notes 9, Macmillan, India, 1981. 
[S] A. Świeçh, Another Approach to the Existence of Value Functions of Stochastic Differential Games, vol. 204, J. Math. Anal. Appl., 1996, pp. 884-897. MR.1422779 (97j:90091)

[SV] D. W. Stroock and S.R.S. Varadhan, Multidimensional Diffusion Processes, SpringerVerlag, Berlin, 1979. MR532498 (81f:60108)

Department of Mathematical Sciences, Florida Institute of Technology, Melbourne, FLORIDA 32901

E-mail address: jkovats@fit.edu 